QUARTERLY OF APPLIED MATHEMATICS

VOLUME LXVII, NUMBER 2

JUNE 2009, PAGES 283-310

S 0033-569X(09)01113-0

Article electronically published on March 20, 2009

\title{
COSINE EFFECT ON SHALLOW WATER EQUATIONS AND MATHEMATICAL PROPERTIES
}

\author{
$\mathrm{BY}$ \\ CARINE LUCAS \\ Laboratoire MAPMO, Université d'Orléans-UFR Sciences, Bât. de Mathématiques-Route de \\ Chartres, BP. 6759, 45067 Orléans cedex 2, France
}

\begin{abstract}
This paper presents a viscous Shallow Water type model with new Coriolis terms, and some limits according to the values of the Rossby and Froude numbers. We prove that the extension to the bidimensional case of the unidimensional results given by [J.-F. Gerbeau, B. Perthame. Discrete Continuous Dynamical Systems, (2001)] including the Coriolis force has to add new terms, omitted up to now, depending on the latitude cosine, when the viscosity is assumed to be of the order of the aspect ratio.

We show that the expressions for the waves are modified, particularly at the equator, as well as the Quasi-Geostrophic and the Lake equations. To conclude, we also study the mathematical properties of these equations.
\end{abstract}

Introduction. In many physical domains, we can come upon thin films such as gravity currents of water (rivers, opened or closed pipes), lava flows or blood in vessels. These thin films are governed by Shallow Water (or Saint-Venant) type equations or Reynolds type equations according to the flow velocity we consider.

This paper details the results announced in [6], where we only indicated the new models and the main existence result through some key points, but without the complete derivations and proofs; we have also added here some numerical experiments which confirm what we said. More precisely, this article concerns a new viscous two dimensional Shallow Water model derived from the three dimensional Navier-Stokes Equations. This model is used to describe vertically averaged flows in three dimensional shallow domains in terms of the horizontal mean velocity field and the depth variation, due to the free surface. The derivation that uses asymptotic analysis is based on the work of GERBEAUPerthame 4 in one dimension, which has been extended to two dimensions by Marche 9. The small parameter of this study is the aspect ratio, denoted by $\varepsilon$, which is the ratio between the characteristic depth and the characteristic length of the domain. We

Received November 1, 2007.

2000 Mathematics Subject Classification. Primary 76M45, 76U05; Secondary 35B40, 35Q35, 46E35.

Key words and phrases. Shallow Water equations, viscosity, Coriolis force, asymptotics, waves, a priori estimates, existence of solutions.

E-mail address: Carine.Lucas@univ-orleans.fr 
first consider the case of a viscosity of order of the aspect ratio. The new point in the corresponding derivation is to remark that, if we want a viscous Shallow Water model at order $\varepsilon$, we cannot take an approximation of the Coriolis force at the first order: the second order produces new terms depending on the cosine of the latitude in the two dimensional Shallow Water model. We also say a word about the case of a viscosity of order one.

Then we look at some physical quantities and the consequences of this new cosine term on the dispersion relation for Poincaré waves and on some equatorial waves. More precisely, we give the equation satisfied by the water height for the linearized rotating Shallow Water equations: it is a Klein-Gordon type equation, modified by the cosine terms. We also study equatorial waves to have the largest influence of the new terms, and we see that they are still trapped in the equatorial waveguide, but their speed has to be changed.

Next we consider two models derived from the Shallow Water theory in which we add our new Coriolis terms: the Quasi-Geostrophic model and the Lake equations. The first one is the result of "filtering out" the fast waves from the rotating barotropic equations. There is a formal analogy between barotropic Quasi-Geostrophic and incompressible flow: in the theory of compressible fluid flows, the incompressible limit is obtained by "filtering out" the "fast" acoustic waves and retaining only the "slow" vorticity modes associated to convection by the fluid. This analogy originally inspired Charney in 1949 when he first formulated the Quasi-Geostrophic equation and thus opened the modern area of numerical weather prediction. To get these equations, we start with the Shallow Water system, where the Rossby and the Froude numbers are supposed to be small, and $\varepsilon$ is fixed. We obtain two new terms that, in some physical cases, are not negligible (see [7).

The Lake equations are obtained by considering the Froude number as a small parameter while the Rossby number and $\varepsilon$ are fixed. These equations may be seen as Shallow Water equations that describe the long-time motion of an incompressible fluid contained in a shallow basin with a slowly spatially varying bottom, a free upper surface and vertical side walls, under the influence of gravity and in the limit of small characteristic velocities and very small surface amplitude.

The last part of this article is devoted to the mathematical properties of these models with new Coriolis terms. The proofs are mainly based on works that have been done without these new terms: the existence of weak solutions for Shallow Water equations relies on new a priori estimates obtained thanks to a new entropy inequality. For the Lake equations, if the water height does not vanish, the proof follows the approach of Levermore, Oliver and Titi in constructing the solutions as the inviscid limit of solutions to a system with artificial viscosity. The degenerate case appeals to the associated Green function. Here, we just have to look at what we can say about our terms to include them in these demonstrations and we get existence of global weak solutions for the viscous two dimensional Shallow Water model. We also obtain existence of global solutions for the Lake equations. 
1. A new viscous shallow water model. The two dimensional Shallow Water system is obtained from three dimensional Navier-Stokes equations in a shallow domain. We look for equations satisfied by the horizontal mean velocity field and the free surface.

We consider 3D Navier-Stokes equations (NSE) for a homogenous fluid:

$$
\begin{gathered}
\partial_{t} U+\operatorname{div}(U \otimes U)=\operatorname{div} \sigma-2 \vec{\Omega} \times U-f, \\
\operatorname{div} U=0,
\end{gathered}
$$

for $(x, z)$ in $\mathbb{T}^{2} \times[b(x), h(t, x)]$, where $U=(u, w) \in \mathbb{R}^{2} \times \mathbb{R}$ is the fluid velocity, $\sigma=$ $-p \mathrm{Id}+2 \mu D(U)$ is the stress tensor, $2 \vec{\Omega} \times U$ is the Coriolis force with $\vec{\Omega}=\Omega(0, \cos \theta, \sin \theta)$, $\theta$ represents the latitude and will be considered as a constant. Finally $f=-g^{t}(0,0,1)$ is the gravity force.

We supplement these equations with the following boundary conditions (see Figure 1):

- at the free surface $z=h(t, x)$ :

We usually neglect atmospheric pressure and take

$$
\sigma n=0,
$$

where $n$ is the unit normal to the surface. Sometimes we can add surface tension effects, that is,

$$
\sigma n=a \kappa n,
$$

where $a$ is the capillarity coefficient and $\kappa$ is the mean curvature.

But in each case we know that the normal velocity in the referential linked to a particle moving on the surface is zero:

$$
\partial_{t} h+u \cdot \nabla_{x} h=w
$$

- at the bottom $z=b(x)$ :

We have Navier type conditions

$$
(\sigma n) \cdot \tau_{1} \tau_{1}+(\sigma n) \cdot \tau_{2} \tau_{2}=k U \cdot \tau_{1} \tau_{1}+k U \cdot \tau_{2} \tau_{2},
$$

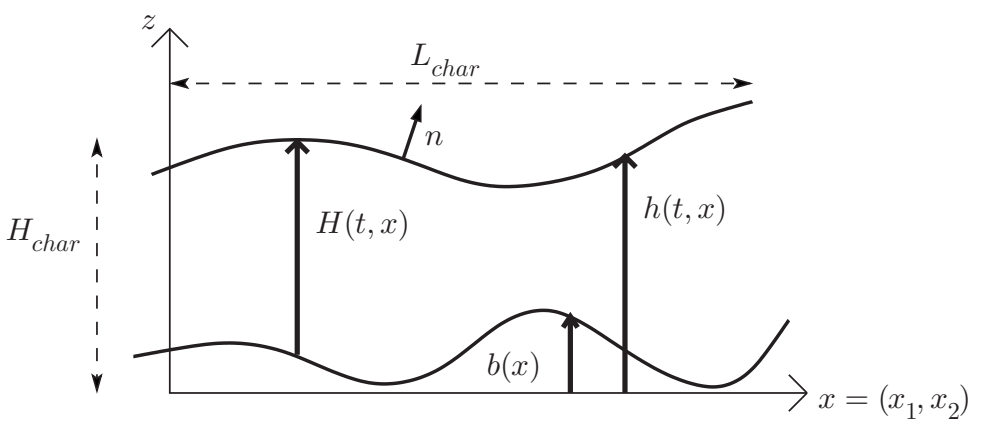

FIG. 1. Notation used for the Shallow Water system 
where $k$ is the friction coefficient and $\left(\tau_{1}, \tau_{2}\right)$ is a basis of the tangential surface. We add the non-penetration condition

$$
-u \cdot \nabla_{x} b+w=0
$$

We can also study turbulent drag terms if we replace $k U$ with $k_{t} H U|U|$ in the previous expression (see [1]).

In what follows we write these equations in a non-dimensional form, replacing $\sigma$ with the expression for the usual Cauchy stress tensor. Next we make an asymptotic development of $U$ and we study its first orders for a viscosity of order $\varepsilon$ (geophysical flows). We get Shallow Water equations by an integration on the water height. We also give the Shallow Water system for thin films, when the viscosity is of order 1.

1.1. Dimensionless Navier-Stokes equations. We write the Navier-Stokes system and the boundary conditions in a non-dimensionalized form, using some characteristic scales specially chosen to get the Shallow Water model.

Let us start with the 3D Navier-Stokes equations for a homogenous fluid, with $\sigma=$ $-p \mathrm{Id}+2 \mu D(U)$, where $\mu$ represents the viscosity and $D(U)$ is the symmetric part of the velocity gradient:

$$
\begin{gathered}
\partial_{t} u+u \cdot \nabla_{x} u+w \partial_{z} u=-\nabla_{x} p+2 \mu \operatorname{div}_{x}\left(D_{x}(u)\right)+\mu \partial_{z}^{2} u+\mu \nabla_{x}\left(\partial_{z} w\right) \\
-2 \Omega \sin \theta u^{\perp}-2 \Omega \cos \theta w e_{1}, \\
\partial_{t} w+u \cdot \nabla_{x} w+w \partial_{z} w=-\partial_{z} p+\mu \partial_{z}\left(\operatorname{div}_{x} u\right)+\mu \Delta_{x} w+2 \mu \partial_{z}^{2} w \\
+2 \Omega \cos \theta u_{1}-g,
\end{gathered}
$$

$\operatorname{div}_{x} u+\partial_{z} w=0$

where the subscript $x$ denotes the horizontal variable, $u_{1}$ and $u_{2}$ are the components of the vector $u, u^{\perp}$ is the vector orthogonal to $u$ defined by ${ }^{t}\left(-u_{2}, u_{1}\right)$ and $e_{1}$ is the unit vector $(1,0)$.

We introduce the following dimensionless variables and numbers:

$$
\begin{array}{ccc}
x=L_{\text {char }} x^{\prime}, & z=H_{\text {char }} z^{\prime} & \text { with } \varepsilon=\frac{H_{\text {char }}}{L_{\text {char }}} \ll 1, \\
u=u_{\text {char }} u^{\prime}, & w=w_{\text {char }} w^{\prime} & \text { with } w_{\text {char }}=\varepsilon u_{\text {char }}, \\
\nu=\frac{\mu}{L_{\text {char }} u_{\text {char }}}, & p=p_{\text {char }} p^{\prime} & \text { with } p_{\text {char }}=u_{\text {char }}^{2}, \\
t=\frac{L_{\text {char }}}{u_{\text {char }}} t^{\prime}, & R o=\frac{u_{\text {char }}}{2 L_{\text {char }} \Omega}, & \text { Fr }=\frac{u_{\text {char }}}{\sqrt{g H_{\text {char }}}},
\end{array}
$$

where $\varepsilon$ is the aspect ratio, Ro the Rossby number, and $F r$ the Froude number. 
We drop the primes to rewrite the 3D NSE:

$$
\begin{aligned}
\partial_{t} u+u \cdot \nabla_{x} u+w \partial_{z} u=- & \nabla_{x} p+2 \nu \operatorname{div}_{x}\left(D_{x}(u)\right)+\frac{\nu}{\varepsilon^{2}} \partial_{z}^{2} u+\nu \nabla_{x}\left(\partial_{z} w\right) \\
& -\frac{\sin \theta}{R o} u^{\perp}-\varepsilon \frac{\cos \theta}{R o} w e_{1}, \\
\partial_{t} w+u \cdot \nabla_{x} w+w \partial_{z} w= & -\frac{1}{\varepsilon^{2}} \partial_{z} p+\frac{\nu}{\varepsilon^{2}} \partial_{z}\left(\operatorname{div}_{x} u\right)+\nu \Delta_{x} w+\frac{2 \nu}{\varepsilon^{2}} \partial_{z}^{2} w \\
& +\frac{1}{\varepsilon} \frac{\cos \theta}{R o} u_{1}-\frac{1}{\varepsilon^{2} F r^{2}}, \\
\operatorname{div}_{x} u+\partial_{z} w=0 . &
\end{aligned}
$$

We also have to change the boundary conditions. First, we replace $\sigma$ with its expression and then change the variables:

- at the free surface $z=h(t, x)$ :

The normal outward vector $n$ is $n=\left(1+\left(\nabla_{x} h\right)^{2}\right)^{-\frac{1}{2}}\left(\begin{array}{c}-\nabla_{x} h \\ 1\end{array}\right)$, and the curvature is given by $\kappa=\operatorname{div}(-n)$.

The horizontal variable $h$ is rescaled as $h=H_{\text {char }} h^{\prime}$ and consequently we have $\kappa=\varepsilon L_{\text {char }}^{-1} \kappa^{\prime}=\varepsilon L_{\text {char }}^{-1} \Delta_{x^{\prime}} h^{\prime}$ up to $O\left(\varepsilon^{3}\right)$. We also define the non-dimensional capillarity coefficient $A=\varepsilon a u_{c h a r}^{-2} L_{c h a r}^{-1}$ to get the dimensionless conditions at the free surface:

$$
\begin{aligned}
& p \nabla_{x} h+A \kappa \nabla_{x} h-2 \nu D_{x}(u) \nabla_{x} h+\nu \nabla_{x} w+\frac{\nu}{\varepsilon^{2}} \partial_{z} u=0, \\
& p+A \kappa-2 \nu \partial_{z} w+\varepsilon^{2} \nu \nabla_{x} w \cdot \nabla_{x} h+\nu \partial_{z} u \cdot \nabla_{x} h=0, \\
& \partial_{t} h+u \cdot \nabla_{x} h=w .
\end{aligned}
$$

- at the bottom $z=b(x)$ :

If we write $b=H_{\text {char }} b^{\prime}$, the non-penetration condition reads

$$
-u \cdot \nabla_{x} b+w=0
$$

The Navier condition is not so simple. We choose the tangential vectors

$$
\tau_{1}=\frac{1}{\left|\nabla_{x} b\right|}\left(\begin{array}{c}
\nabla_{x}^{\perp} b \\
0
\end{array}\right) \quad \text { and } \quad \tau_{2}=\frac{1}{\sqrt{\left|\nabla_{x} b\right|^{2}+\left|\nabla_{x} b\right|^{4}}}\left(\begin{array}{c}
-\nabla_{x} b \\
-\left|\nabla_{x} b\right|^{2}
\end{array}\right)
$$

We define $K=k u_{\text {char }}^{-1}, K_{t}=k_{t} L_{\text {char }}$ and we get a complex expression we do not detail here. We perform some approximations in the following and then give a simplified equality obtained from this expression.

1.2. Hydrostatic approximation. We now use the hydrostatic approximation, that is, we suppose $\varepsilon$ to be small. We only keep the first two orders in Equation (1.2) and we also drop terms in the boundary conditions. 
We are led to study the system:

$$
\begin{gathered}
\partial_{t} u+u \cdot \nabla_{x} u+w \partial_{z} u=-\nabla_{x} p+2 \nu \operatorname{div}_{x}\left(D_{x}(u)\right)+\frac{\nu}{\varepsilon^{2}} \partial_{z}^{2} u+\nu \nabla_{x}\left(\partial_{z} w\right) \\
-\frac{\sin \theta}{R o} u^{\perp}-\varepsilon \frac{\cos \theta}{R o} w e_{1}, \\
\partial_{z} p=\nu \partial_{z}\left(\operatorname{div}_{x} u\right)+2 \nu \partial_{z}^{2} w-\frac{1}{F r^{2}}+\varepsilon \frac{\cos \theta}{R o} u_{1}, \\
\operatorname{div}_{x} u+\partial_{z} w=0 .
\end{gathered}
$$

We can simplify the Navier condition

$$
\begin{aligned}
2 \varepsilon \nu D_{x}(u) \cdot \nabla_{x} b-\varepsilon \nu & \left(\frac{1}{\varepsilon^{2}} \partial_{z} u+\nabla_{x} w\right) \\
& =-K u-\varepsilon K_{t} H u|u|+2 \varepsilon \nu \partial_{z} w \nabla_{x} b+\varepsilon \nu\left(\nabla_{x} b \cdot \partial_{z} u\right) \nabla_{x} b .
\end{aligned}
$$

At the free surface, Equation (1.6) is not modified, but we can rewrite (1.4) as follows:

$$
\nu \partial_{z} u=-\varepsilon^{2}\left(p \nabla_{x} h+A \kappa \nabla_{x} h-2 \nu D_{x}(u) \nabla_{x} h+\nu \nabla_{x} w\right) \text { for } z=h(x, t),
$$

and we replace it in Equation (1.5):

$$
\begin{aligned}
p+A \kappa-2 \nu \partial_{z} w & =-\varepsilon^{2} \nu \nabla_{x} w \cdot \nabla_{x} h-\nu \partial_{z} u \cdot \nabla_{x} h \\
& =\varepsilon^{2}\left(p \nabla_{x} h+A \kappa \nabla_{x} h-2 \nu D_{x}(u) \nabla_{x} h\right) \quad \text { for } z=h(x, t) .
\end{aligned}
$$

As $\varepsilon$ tends to zero, we get

$$
p+A \kappa-2 \nu \partial_{z} w=0 \quad \text { for } z=h(x, t) .
$$

We integrate the vertical derivative of the pressure (given by Equation (1.9) ) from $h$ to $z$, with $z$ between $b$ and $h$. The value of $p$ at the free surface is given by (1.12) and with the divergence free condition (1.10) we find the pressure at order $\varepsilon$ :

$$
p(t, x, z)=\frac{1}{F r^{2}}(h(t, x)-z)-A \kappa-\nu\left(\operatorname{div}_{x} u\right)_{\left.\right|_{z=h(t, x)}}-\nu \operatorname{div}_{x} u+\varepsilon \frac{\cos \theta}{R o} \int_{h}^{z} u_{1} .
$$

As we are looking for equations on the mean velocity and on the evolution of the free surface, we first integrate the momentum equation (1.8) over the water height (between $z=b(x)$ and $z=h(t, x))$.

We apply the Leibniz formula and get:

$$
\begin{gathered}
\partial_{t} \int_{b}^{h} u-\partial_{t} h u_{\left.\right|_{z=h}}+\operatorname{div}_{x} \int_{b}^{h}(u \otimes u)-\left(\left(u \cdot \nabla_{x} h\right) u\right)_{\left.\right|_{z=h}}+\left(\left(u \cdot \nabla_{x} b\right) u\right)_{\left.\right|_{z=b}} \\
+(u w)_{\left.\right|_{z=h}}-(u w)_{\left.\right|_{z=b}}+\nabla_{x} \int_{b}^{h} p=\nabla_{x} h p_{\left.\right|_{z=h}}-\nabla_{x} b p_{\left.\right|_{z=b}} \\
+2 \nu \operatorname{div}_{x} \int_{b}^{h} D_{x}(u)-2 \nu D_{x}(u)_{\left.\right|_{z=h}} \nabla_{x} h+2 \nu D_{x}(u)_{\left.\right|_{z=b}} \nabla_{x} b+\frac{\nu}{\varepsilon^{2}} \partial_{z} u_{\left.\right|_{z=h}} \\
-\frac{\nu}{\varepsilon^{2}} \partial_{z} u_{\left.\right|_{z=b}}+\nu \nabla_{x} w_{\left.\right|_{z=h}}-\nu \nabla_{x} w_{\left.\right|_{z=b}}-\frac{\sin \theta}{R o} \int_{b}^{h} u^{\perp}-\varepsilon \frac{\cos \theta}{R o} \int_{b}^{h} w e_{1} .
\end{gathered}
$$


Then, we use the boundary conditions (1.4), (1.6), (1.7), (1.11) to simplify the expressions at the surface and at the bottom, and we get the integrated momentum equation:

$$
\begin{aligned}
& \partial_{t} \int_{b}^{h} u+\operatorname{div}_{x} \int_{b}^{h}(u \otimes u)+\nabla_{x} \int_{b}^{h} p \\
&=-A \kappa \nabla_{x} h-\nabla_{x} b p_{\left.\right|_{z=b}}-\left(\frac{K}{\varepsilon} u+K_{t} H u|u|\right)_{\mid z=b}+2 \nu \operatorname{div}_{x} \int_{b}^{h} D_{x}(u) \\
& \quad+\nu \nabla_{x} b\left(2 \partial_{z} w+\nabla_{x} b \cdot \partial_{z} u\right)_{\left.\right|_{z=b}}-\frac{\sin \theta}{R o} \int_{b}^{h} u^{\perp}-\varepsilon \frac{\cos \theta}{R o} \int_{b}^{h} w e_{1},
\end{aligned}
$$

with a new Coriolis term (the last one).

We also want the evolution of the free surface: we integrate the divergence free equation (1.10) from the bottom to the surface, and use the Leibniz formula again and the conditions at the surface (1.6) and at the bottom (1.7) to find:

$$
\partial_{t} h(t, x)+\operatorname{div}_{x} \int_{b(x)}^{h(t, x)} u=0 .
$$

In the sequel we fix the values of $\nu, K$ and $K_{t}$ with respect to $\varepsilon$ and we study the integrated momentum equation (1.14) and the free surface equation (1.15) when we approximate $u$ at the first order and at the second order.

1.3. Shallow Water system with a viscosity of order $\varepsilon$. We have already done the main assumption to get the Shallow Water system, that is, the depth is small compared to the length of the domain. Now, we make some hypotheses on the physical quantities.

In this part, we consider $\nu, A, K$ and $K_{t}$ of order $\varepsilon: \nu=\varepsilon \nu_{0}, A=\varepsilon A_{0}, K=\varepsilon K_{0}$ and $K_{t}=\varepsilon K_{0 t}$.

We develop $u, w, H, p, b$ in powers of $\varepsilon$, that is, $u=u^{0}+\varepsilon u^{1}+\varepsilon^{2} u^{2}+\ldots$ (and so on) with $H(t, x)=h(t, x)-b(x)$.

1.3.1. At the first order. We want an expression of the first order of the velocity $u^{0}$. We use the horizontal momentum equation (1.8) and the boundary conditions (1.4) and (1.11). We obtain

$$
\partial_{z}^{2} u=O(\varepsilon), \quad\left(\partial_{z} u\right)_{\left.\right|_{z=b}}=O(\varepsilon), \quad\left(\partial_{z} u\right)_{\left.\right|_{z=h}}=O(\varepsilon)
$$

which means that at the first order $u$ does not depend on $z: u^{0}(t, x, z)=u^{0}(t, x)$, still unknown. As we are looking for the dynamics on $u^{0}$, we study the previous equations at the first order. We can already rewrite the evolution equation (1.15) as

$$
\partial_{t} H^{0}+\operatorname{div}_{x}\left(H^{0} u^{0}\right)=0 .
$$

Then we have the expression of $p$ at the first order with (1.13):

$$
p^{0}(t, x, z)=F r^{-2}(h-z) .
$$


We replace this value in the integrated momentum equation (1.14) and we find the equality:

$$
\begin{aligned}
\partial_{t}\left(H^{0} u^{0}\right)+\operatorname{div}_{x}\left(H^{0} u^{0} \otimes u^{0}\right)+\frac{1}{2 F r^{2}} & \nabla_{x}\left(H^{0}\right)^{2} \\
& =-\frac{1}{F r^{2}} H^{0} \nabla_{x} b^{0}-K_{0} u^{0}-\frac{\sin \theta}{R o} H^{0} u^{0^{\perp} .}
\end{aligned}
$$

Equations (1.16) -(1.17) form the Shallow Water system at the first order in non-dimensional variables.

If we go back to dimensional variables we have the Shallow Water system at the first order for a viscosity of order $\varepsilon$ :

$$
\begin{aligned}
& \partial_{t} H+\operatorname{div}_{x}(H u)=0, \\
& \partial_{t}(H u)+\operatorname{div}_{x}(H u \otimes u)+\frac{g}{2} \nabla_{x} H^{2}=-g H \nabla_{x} b-k u-2 \Omega \sin \theta H u^{\perp} .
\end{aligned}
$$

These equations are the classical generalization to the 2D case of the ones given by A. J.-C. de Saint-Venant in [12. But there are no viscosity effects in this system: following [4, we are led to study the equations we obtain at the second order to see the viscous terms in our Shallow Water system.

1.3.2. At the second order. We study here the equations we can obtain at the second order because we want a viscous system.

We will denote by a bold letter the approximation of the variables at order $\varepsilon$ (for example, $\boldsymbol{u}^{\mathbf{1}}=u^{0}+\varepsilon u^{1}$ ) and by a bar the mean value on the water height:

$$
\bar{u}:=\frac{1}{H(t, x)} \int_{b}^{h} u d z .
$$

We rewrite the divergence condition at the second order:

$$
\partial_{t} \boldsymbol{H}^{\mathbf{1}}+\operatorname{div}_{x}\left(\boldsymbol{H}^{\mathbf{1}} \overline{\boldsymbol{u}}^{\mathbf{1}}\right)=O\left(\varepsilon^{2}\right) .
$$

As before, we rewrite the momentum equation (1.8) but at order $\varepsilon$, and, with the Shallow Water system at the first order (1.16) (1.17), we find an expression of the second derivative of $u$ :

$$
\frac{\nu}{\varepsilon^{2}} \partial_{z}^{2} u=-\frac{K_{0}}{H^{0}} u^{0}+O(\varepsilon) .
$$

We integrate this equality from $b$ to $z$ (for $z$ between $b(x)$ and $h(t, x)$ ) with the boundary condition (1.11). We integrate it again from $b$ to $z$ to find an approximation of $u$ at the second order:

$$
\begin{aligned}
u & =\boldsymbol{u}_{\left.\right|_{z=b}}+\varepsilon \frac{K_{0}}{\nu_{0}} u^{0} \int_{b}^{z}\left(1-\frac{s-b^{0}}{H^{0}}\right) d s+O\left(\varepsilon^{2}\right) \\
& =\boldsymbol{u}_{\left.\right|_{z=b}}\left(1+\varepsilon \frac{K_{0}}{\nu_{0}}\left(z-b^{0}\right)\left(1-\frac{z-b^{0}}{2 H^{0}}\right)\right)+O\left(\varepsilon^{2}\right) .
\end{aligned}
$$

With this expression, we can compute the mean value of $u$ :

$$
\bar{u}=u_{\left.\right|_{z=b}}\left(1+\varepsilon \frac{K_{0}}{\nu_{0}} \frac{H^{0}}{3}\right)+O\left(\varepsilon^{2}\right) .
$$

One can notice that the mean value of $u^{2}$ is equal to the square of the mean value of $u$ up to $O\left(\varepsilon^{2}\right)$. We have the same property for $u \otimes u$. This is used in the sequel. 
We also have the value of $p$ at the second order with Equation (1.13) and we can replace it in the integrated momentum equation (1.14). Using again the divergence free condition (1.10) to express $w^{0}$ as a function of $h^{0}$ and $u^{0}$, we get

$$
\begin{aligned}
\partial_{t}\left(\boldsymbol{H}^{\mathbf{1}} \bar{u}\right)+\operatorname{div}_{x}\left(\boldsymbol{H}^{\mathbf{1}} \bar{u} \otimes \bar{u}\right)+\frac{1}{2 F r^{2}} \nabla_{x}\left(\boldsymbol{H}^{\mathbf{1}}\right)^{2}-\varepsilon A_{0} \nabla_{x}\left(H^{0} \Delta_{x} h^{0}\right) \\
-2 \varepsilon \nu_{0} \nabla_{x}\left(H^{0} \operatorname{div}_{x} u^{0}\right)-\varepsilon \frac{\cos \theta}{2 R o} \nabla_{x}\left(u_{1}^{0}\left(H^{0}\right)^{2}\right)=-\left(K_{0} \boldsymbol{u}^{\mathbf{1}}+\varepsilon K_{0 t} H^{0} \boldsymbol{u}^{\mathbf{1}}\left|\boldsymbol{u}^{\mathbf{1}}\right|\right)_{\left.\right|_{z=b}} \\
-\nabla_{x} b\left(\frac{\boldsymbol{H}^{\mathbf{1}}}{F r^{2}}-\varepsilon A_{0} \Delta_{x} h^{0}-2 \varepsilon \nu_{0} \operatorname{div}_{x} u^{0}-\varepsilon \frac{\cos \theta}{R o} u_{1}^{0} H^{0}\right)-\varepsilon A_{0} \Delta_{x} h^{0} \nabla_{x} h^{0} \\
+\varepsilon \nu_{0} \nabla_{x} b^{0}\left(2 \partial_{z} w^{0}+\nabla_{x} b^{0} \cdot \partial_{z} u^{0}\right)_{\left.\right|_{z=b}}+2 \varepsilon \nu_{0} \operatorname{div}_{x}\left(H^{0} D_{x} u^{0}\right) \\
\quad-\frac{\sin \theta}{R o} \boldsymbol{H}^{\mathbf{1}} \bar{u}^{\perp}+\varepsilon \frac{\cos \theta}{2 R o}\left(H^{0}\right)^{2} e_{1} \operatorname{div}_{x} u^{0}-\varepsilon \frac{\cos \theta}{R o} H^{0} e_{1} \nabla_{x} b^{0} \cdot u^{0}+O\left(\varepsilon^{2}\right) .
\end{aligned}
$$

The velocity at the bottom $u_{\left.\right|_{z=b}}$ is given by

$$
\boldsymbol{u}_{\mid z=b}^{\mathbf{1}}=\bar{u}\left(1+\frac{\varepsilon K_{0}}{\nu_{0}} \frac{H^{0}}{3}\right)^{-1}+O\left(\varepsilon^{2}\right)
$$

which leads to:

$$
\begin{aligned}
\partial_{t}\left(\boldsymbol{H}^{\mathbf{1}} \bar{u}\right) & +\operatorname{div}_{x}\left(\boldsymbol{H}^{\mathbf{1}} \bar{u} \otimes \bar{u}\right)+\frac{1}{2 F r^{2}} \nabla_{x}\left(\boldsymbol{H}^{\mathbf{1}}\right)^{2}=-K_{0} \bar{u}\left(1+\frac{\varepsilon K_{0}}{\nu_{0}} \frac{\boldsymbol{H}^{\mathbf{1}}}{3}\right)^{-1} \\
& -\varepsilon K_{0 t} \boldsymbol{H}^{\mathbf{1}}\left(1+\frac{\varepsilon K_{0}}{\nu_{0}} \frac{\boldsymbol{H}^{\mathbf{1}}}{3}\right)^{-2} \bar{u}|\bar{u}|+\varepsilon A_{0} \boldsymbol{H}^{\mathbf{1}} \nabla_{x} \Delta_{x} \boldsymbol{H}^{\mathbf{1}} \\
& +\varepsilon A_{0} \boldsymbol{H}^{\mathbf{1}} \nabla_{x} \Delta_{x} b+2 \varepsilon \nu_{0} \nabla_{x}\left(\boldsymbol{H}^{\mathbf{1}} \operatorname{div}_{x} \bar{u}\right)+2 \varepsilon \nu_{0} \operatorname{div}_{x}\left(\boldsymbol{H}^{\mathbf{1}} D_{x} \bar{u}\right) \\
& +\varepsilon \frac{\cos \theta}{2 R o} \nabla_{x}\left(\bar{u}_{1}\left(\boldsymbol{H}^{\mathbf{1}}\right)^{2}\right)+\varepsilon \frac{\cos \theta}{2 R o}\left(\boldsymbol{H}^{\mathbf{1}}\right)^{2} e_{1} \operatorname{div}_{x} \bar{u}-\varepsilon \frac{\cos \theta}{R o} \boldsymbol{H}^{\mathbf{1}} e_{1} \nabla_{x} b \cdot \bar{u} \\
& -\frac{\sin \theta}{R o} \boldsymbol{H}^{\mathbf{1}} \bar{u}^{\perp}-\nabla_{x} b\left(\frac{\boldsymbol{H}^{\mathbf{1}}}{F r^{2}}-\varepsilon \frac{\cos \theta}{R o} \bar{u}_{1} \boldsymbol{H}^{\mathbf{1}}\right)+O\left(\varepsilon^{2}\right) .
\end{aligned}
$$

Equations (1.20)-(1.21) form the Shallow Water system at the second order in nondimensional variables, with new cosine terms.

Finally, let us go back to the dimensional form to get the viscous Shallow Water system at the second order:

$$
\begin{aligned}
& \partial_{t} H+\operatorname{div}_{x}(H u)=0, \\
& \partial_{t}(H u)+\operatorname{div}_{x}(H u \otimes u)+\frac{g}{2} \nabla_{x} H^{2}=-\alpha_{0}(H) u-\alpha_{1}(H) H u|u| \\
& \quad+a H \nabla_{x} \Delta_{x} H+2 \mu \nabla_{x}\left(H \operatorname{div}_{x} u\right)+2 \mu \operatorname{div}_{x}\left(H D_{x} u\right) \\
& \quad+\Omega \cos \theta \nabla_{x}\left(u_{1} H^{2}\right)+\Omega \cos \theta H^{2} e_{1} \operatorname{div}_{x} u-2 \Omega \sin \theta H u^{\perp} \\
& \quad-2 \Omega \cos \theta H e_{1} \nabla_{x} b \cdot u+2 \Omega \cos \theta u_{1} H \nabla_{x} b+a H \nabla_{x} \Delta_{x} b-g H \nabla_{x} b,
\end{aligned}
$$

where

$$
\alpha_{0}(H)=\frac{k}{1+\frac{k H}{3 \mu}}, \quad \alpha_{1}(H)=\frac{k_{t}}{\left(1+\frac{k H}{3 \mu}\right)^{2}}
$$


We now have the viscous terms we could not find at the first order. We remark that the cosine part of the Coriolis force must then be taken into account. We briefly consider another physical case, where the viscosity is of order one.

1.4. If the viscosity is of order one. We have looked at a viscosity of order $\varepsilon$ in the previous part and found that going to the second order was necessary to see viscous terms. Here, we keep all the parameters unchanged but we modify the viscosity.

If the viscosity is of order one, we find again:

$$
\partial_{z}^{2} u(t, x, z)=O(\varepsilon), \quad \partial_{z} u(t, x, 0)=O(\varepsilon), \quad \partial_{z} u(t, x, h(t, x))=O(\varepsilon) ;
$$

that is, the main order of the velocity does not depend on $z: u^{0}(t, x, z)=u^{0}(t, x, 0)$, which is unknown.

As before, we get Equation (1.16) and $p^{(0)}$.

The integration of the horizontal momentum equation at the first order leads to

$$
\begin{aligned}
\partial_{t}(H u)+ & \operatorname{div}_{x}(H u \otimes u)+\frac{g}{2} \nabla_{x} H^{2}=-2 \Omega \sin \theta H u^{\perp}+a H \nabla_{x} \Delta_{x} b \\
& -g H \nabla_{x} b-k u+a H \nabla_{x} \Delta_{x} H+2 \mu \nabla_{x}\left(H \operatorname{div}_{x} u\right)+2 \mu \operatorname{div}_{x}\left(H D_{x} u\right) .
\end{aligned}
$$

Note that we have viscous terms in this equality, which is obtained at the first order; there is no need for a correcting term on $u$ at the second order to see viscosity effects.

We obtained Shallow Water models with viscous terms. When the viscosity is of order one, the first order of the system is sufficient, and the Coriolis terms reduce to the usual ones. But when the viscosity is of order $\varepsilon$, it is necessary to go to the second order, and then we must take into account the cosine effect of the Coriolis terms: this creates a new model.

As our new terms are linked to the cosine of the latitude, the first idea is to study this new model where the cosine plays a great role, that is, near the equator. Therefore, in the following, we describe various waves and we emphasize equatorial waves obtained when the tangential projection of the Coriolis force vanishes.

2. Waves for rotating shallow water equations. This study is based on $[8$, where the results are obtained without the cosine terms. We consider the non-dimensional Shallow Water equations at the second order (Equations (1.20)-(1.21)), without viscosity, surface tension or turbulent drag.

This section is divided into three parts: first, we write the potential-vorticity conservation for the new rotating Shallow Water equations. Then we study the linearized equations and give the Poincaré waves and their dispersion relation. The last results are about equatorial waves: at the equator, the Coriolis force is not complete; the tangential part vanishes. As a physical consequence of this simplification, we see that the tropics behave as a waveguide, and we prove that the wave speed depends on the cosine term.

2.1. Potential vorticity conservation. The vorticity, defined by $\omega=\operatorname{curl} u$, satisfies the following equation:

$$
\begin{aligned}
\partial_{t} \omega+u \cdot \nabla \omega+ & \omega \operatorname{div} u \\
& =-\frac{\sin \theta}{R o} \operatorname{div} u+\varepsilon \frac{\cos \theta}{2 R o} \operatorname{curl}\left(H \nabla u_{1}+2 u_{1} \nabla h+H e_{1} \operatorname{div} u-2 e_{1} \nabla b \cdot u\right) .
\end{aligned}
$$


The water height equation allows us to write:

$$
\begin{aligned}
\operatorname{curl}\left(H \nabla u_{1}+2 u_{1} \nabla h\right. & \left.+H e_{1} \operatorname{div} u-2 e_{1} \nabla b \cdot u\right) \\
& =\partial_{x_{2}} H \operatorname{div} u+u \cdot \nabla \partial_{x_{2}} H+2 \partial_{x_{2}} b \operatorname{div} u+2 u \cdot \nabla \partial_{x_{2}} b+\partial_{t} \partial_{x_{2}} H,
\end{aligned}
$$

and, replacing it in the whole equation, we find

$$
\begin{aligned}
\left(\partial_{t}+u \cdot \nabla\right)\left(\omega+\frac{\sin \theta}{R o}-\varepsilon \frac{\cos \theta}{2 R o} \partial_{x_{2}}(H\right. & +2 b)) \\
& +\left(\omega+\frac{\sin \theta}{R o}-\varepsilon \frac{\cos \theta}{2 R o} \partial_{x_{2}}(H+2 b)\right) \operatorname{div} u=0 .
\end{aligned}
$$

We multiply this expression by $H$ and add the water height conservation multiplied by $\left(-2 R o \omega-2 \sin \theta+\varepsilon \cos \theta \partial_{x_{2}}(H+2 b)\right) /(2 R o)$. We divide the result by $H^{2}$ and get

$$
\left(\partial_{t}+u \cdot \nabla\right)\left(\frac{2 R o \omega+2 \sin \theta-\varepsilon \cos \theta \partial_{x_{2}}(H+2 b)}{2 R o H}\right)=0 .
$$

This relation expresses the conservation of a new potential vorticity defined by

$$
\left(2 R o \omega+2 \sin \theta-\varepsilon \cos \theta \partial_{x_{\mathbf{2}}}(\boldsymbol{H}+\mathbf{2 b})\right) /(2 R o H) .
$$

Compared with the usual definition (see 8$]$ for example), we find a new term, the bold one, depending on the cosine of the latitude.

2.2. Linear theory and Poincaré waves. In order to derive the linearized rotating Shallow Water equations, we consider the non-dimensional Shallow Water system at the second order, with a flat bottom. We look at small perturbations around the constant background state $\left(H=H^{c}, u=0\right)$ of the form:

$$
H=H^{c}+\delta^{p} H^{p}, \quad u=\delta^{p} u^{p}, \quad \text { with } \delta^{p} \ll 1,
$$

and we keep the first order in $\delta^{p}$ :

$$
\begin{aligned}
& \partial_{t} H^{p}+H^{c} \operatorname{div} u^{p}=0, \\
& \partial_{t} u^{p}+\frac{1}{F r^{2}} \nabla H^{p}=\varepsilon \frac{\cos \theta}{2 R o} H^{c} \nabla u^{p}+\varepsilon \frac{\cos \theta}{2 R o} H^{c} e_{1} \operatorname{div} u^{p}-\frac{\sin \theta}{R o} u^{p \perp} .
\end{aligned}
$$

From these equations, we can derive Poincaré waves, also called inertio-gravity waves. We differentiate in time Equation (2.1), replace the term $\operatorname{div} \partial_{t} u$ using Equation (2.2), and derive again in time. We get

$$
\begin{aligned}
\partial_{t}^{3} H^{p}=\frac{1}{F r^{2}} H^{c} \Delta\left(\partial_{t} H^{p}\right) & \\
& -\varepsilon H^{c 2} \frac{\cos \theta}{2 R o} \partial_{t} \Delta u_{1}^{p}-\varepsilon H^{c 2} \frac{\cos \theta}{2 R o} \partial_{x_{1}} \partial_{t} \operatorname{div} u^{p}-H^{c} \frac{\sin \theta}{R o} \partial_{t} \operatorname{curl} u^{p} .
\end{aligned}
$$

If we define $\omega=\delta^{p} \omega^{p}=\delta^{p} \operatorname{curl} u^{p}$, the potential vorticity conservation can be linearized as

$$
\partial_{t} \omega^{p}-\frac{\sin \theta}{H^{c} R o} \partial_{t} H^{p}-\varepsilon \frac{\cos \theta}{2 R o} \partial_{x_{2}} \partial_{t} H^{p}=0
$$


We also have from Equations (2.1) and (2.2) the equalities

$$
\begin{aligned}
& \partial_{x_{1}} \partial_{t} \operatorname{div} u^{p}=-\frac{1}{H^{c}} \partial_{x_{1}} \partial_{t}\left(\partial_{t} H^{p}\right), \\
& \begin{aligned}
\partial_{t} \Delta u_{1}^{p} & =\partial_{t}\left(\partial_{x_{1}} \operatorname{div} u^{p}-\partial_{x_{2}} \omega\right) \\
& =-\frac{1}{H^{c}} \partial_{x_{1}} \partial_{t}\left(\partial_{t} H^{p}\right)-\varepsilon \frac{\cos \theta}{2 R o} \partial_{x_{2}}^{2}\left(\partial_{t} H^{p}\right)-\frac{\sin \theta}{H^{c} R O} \partial_{x_{2}}\left(\partial_{t} H^{p}\right),
\end{aligned}
\end{aligned}
$$

which give

$$
\begin{aligned}
\left(\partial_{t}-\varepsilon H^{c} \frac{\cos \theta}{2 R o} \partial_{x_{1}}\right)^{2}( & \left.\partial_{t} H^{p}\right) \\
& =\left(\frac{1}{F r^{2}} H^{c}+\left(\varepsilon H^{c} \frac{\cos \theta}{2 R o}\right)^{2}\right) \Delta\left(\partial_{t} H^{p}\right)-\left(\frac{\sin \theta}{R o}\right)^{2}\left(\partial_{t} H^{p}\right) .
\end{aligned}
$$

We can also define a new function depending on new variables:

$$
H^{p}\left(x_{1}, x_{2}, t\right)=G^{p}\left(y_{1}, y_{2}, s\right)=G^{p}\left(x_{1}+\varepsilon H^{c} \frac{\cos \theta}{2 R o} t, x_{2}, t\right) .
$$

Then, the function $G^{\boldsymbol{p}}\left(y_{1}, y_{2}, s\right)=\left(\varepsilon H^{c} \cos \theta /(2 R o) \partial_{y_{1}}+\partial_{s}\right) G^{p}\left(y_{1}, y_{2}, s\right)$ satisfies the Klein-Gordon equation:

$$
\partial_{s}^{2} G^{\boldsymbol{p}}=\left(\frac{1}{F r^{2}} H^{c}+\left(\varepsilon H^{c} \frac{\cos \theta}{2 R o}\right)^{2}\right)\left(\partial_{y_{1}}^{2}+\partial_{y_{2}}^{2}\right) G^{\boldsymbol{p}}-\left(\frac{\sin \theta}{R o}\right)^{2} G^{p} .
$$

To get the dispersion relation, we write $H^{p}(x, t)=H_{0} \exp \left(i\left(k_{1} x_{1}+k_{2} x_{2}-\tilde{W} t\right)\right)$ or $G^{p}(y, s)=G_{0} \exp \left(i\left(K_{1} y_{1}+K_{2} y_{2}-W s\right)\right)$, with $H_{0}=G_{0}, K_{1}=k_{1}, K_{2}=k_{2}$ and $W=\tilde{W}+\varepsilon H^{c} \cos \theta k_{1} /(2 R o)$. We obtain:

$$
\tilde{W}(k)=-\varepsilon H^{c} \frac{\cos \theta}{2 R o} k_{1} \pm \sqrt{\left(\frac{1}{F r 2} H^{c}+\left(\varepsilon H^{c} \frac{\cos \theta}{2 R o}\right)^{2}\right)|k|^{2}+\left(\frac{\sin \theta}{R o}\right)^{2}}
$$

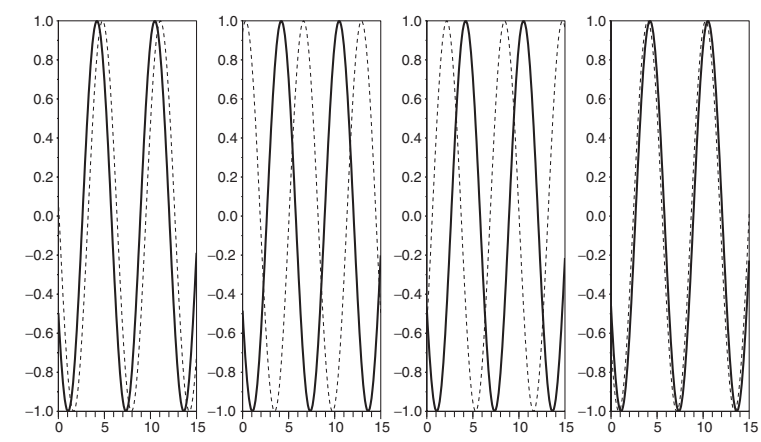

FIG. 2. Wave with (solid line) and without (dotted line) cosine effect for a latitude equal to $30^{\circ}$, at times $10 \mathrm{~s}, 40 \mathrm{~s}, 70 \mathrm{~s}$ and $100 \mathrm{~s}$. 


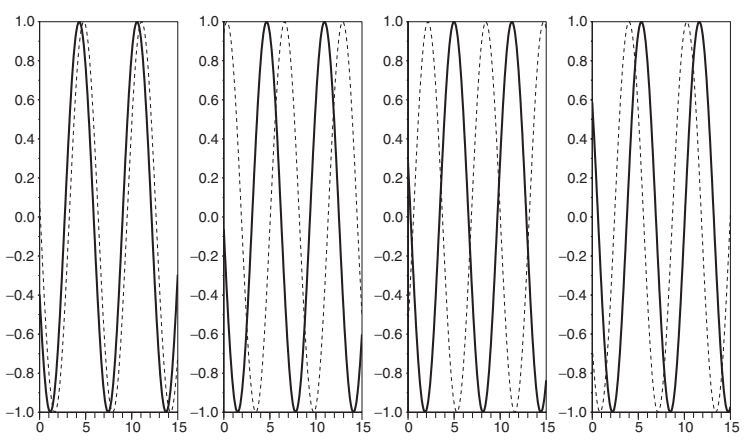

FIG. 3. Wave with (solid line) and without (dotted line) cosine effect for a latitude equal to $45^{\circ}$, at times $10 \mathrm{~s}, 40 \mathrm{~s}, 70 \mathrm{~s}$ and $100 \mathrm{~s}$.

or

$$
W(K)= \pm \sqrt{\left(\frac{1}{F r 2} H^{c}+\left(\varepsilon H^{c} \frac{\cos \theta}{2 R o}\right)^{2}\right)|k|^{2}+\left(\frac{\sin \theta}{R o}\right)^{2}} .
$$

If we want the dimensional results, we just have to replace $\varepsilon H^{c} \cos \theta /(2 R o)$ by $H^{c} \Omega \cos \theta$, $\sin \theta / R o$ by $2 \Omega \sin \theta$ and $1 / F r^{2}$ by $g$. Then we can look at the difference due to the cosine effect: we plot $H^{p}$ in the dimensional case, with $k_{1}=1, k_{2}=0, H^{c}=1000 \mathrm{~m}$, $\Omega=7 * 10^{-5} \mathrm{~s}^{-1}, g=9.81 \mathrm{~ms}^{-2}$ and at the latitude $\theta=\pi / 6$ or $\pi / 4$, with and without the new terms. We see, Figures 2 and 3 , that the discrepancy between the two curves is not negligible.

2.3. Equatorial waves. To see the effects of the cosine terms, we can study waves where the latitude vanishes, near the equator. We start with the linearized equations to obtain Kelvin waves and mixed Rossby-gravity waves.

Kelvin waves are an important part of the observational record of the equatorial ocean. We prove that they are trapped in the equatorial wave guide and that their speed depends on the cosine term. We study flows which are parallel to the equator, that is, with $u_{2}=0$, and we use the $\beta$-plane approximation in the linearized equations (2.1)-(2.2) to get:

$$
\begin{aligned}
& \beta x_{2} u_{1}^{p}=-\frac{1}{F r^{2}} \partial_{x_{2}} H^{p}+\varepsilon \frac{1}{2 R o} H^{c} \partial_{x_{2}} u_{1}^{p}, \\
& \partial_{t} u_{1}^{p}+\frac{1}{F r^{2}} \partial_{x_{1}} H^{p}=\varepsilon \frac{1}{R o} H^{c} \partial_{x_{1}} u_{1}^{p}, \\
& \partial_{t} H^{p}+H^{c} \partial_{x_{1}} u_{1}^{p}=0 .
\end{aligned}
$$

We introduce the characteristic variables:

$$
q=\frac{c_{2}}{H^{c}} H^{p}+u_{1}^{p}, \quad r=\frac{c_{1}}{H^{c}} H^{p}-u_{1}^{p},
$$

which satisfy

$$
\frac{\partial q}{\partial t}+c_{1} \frac{\partial q}{\partial x_{1}}=0 \quad(\text { eastward }), \quad \text { and } \quad \frac{\partial r}{\partial t}-c_{2} \frac{\partial r}{\partial x_{1}}=0 \quad \text { (westward) }
$$


where the speeds are defined by

$$
c_{1}=\sqrt{\frac{H^{c}}{F r^{2}}+\varepsilon^{2} \frac{H^{c^{2}}}{(2 R o)^{2}}}-\varepsilon \frac{1}{2 R o} H^{c}, \quad c_{2}=\sqrt{\frac{H^{c}}{F r^{2}}+\varepsilon^{2} \frac{H^{c^{2}}}{(2 R o)^{2}}}+\varepsilon \frac{1}{2 R o} H^{c} .
$$

These two waves should be combined to find a general solution to our problem. But we must seek their structure in the second space variable.

For the eastward group $(r \equiv 0)$, if we write $q=G\left(x_{2}\right) q\left(x_{1}-c_{1} t\right)$ we find, with previous equations, an expression for $G$ :

$$
G\left(x_{2}\right)=G_{0} \exp \left(\frac{-\beta}{c_{1}+c_{2}} x_{2}^{2}\right),
$$

which means that these waves are trapped in the equatorial waveguide.

For the westward group $(q \equiv 0)$, we find an unphysical solution.

Then we conclude that Kelvin waves only propagate in the eastward direction, with the speed $c_{1}$, are non-dispersive and are trapped in the equatorial waveguide.

We can also look at mixed Rossby-gravity waves, or Yanai waves. We write $u_{1}^{p}$ and $H^{p}$ in terms of the characteristic variables $q$ and $r$, and we modify the linearized Shallow Water equations:

$$
\begin{array}{r}
\frac{\partial q}{\partial t}+c_{1} \frac{\partial q}{\partial x_{1}}+\left(\frac{c_{1}+c_{2}}{2}\right) \frac{\partial u_{2}^{p}}{\partial x_{2}}-\beta x_{2} u_{2}^{p}=0, \\
\frac{\partial r}{\partial t}-c_{2} \frac{\partial r}{\partial x_{1}}+\left(\frac{c_{1}+c_{2}}{2}\right) \frac{\partial u_{2}^{p}}{\partial x_{2}}+\beta x_{2} u_{2}^{p}=0, \\
\frac{\partial u_{2}^{p}}{\partial t}+\frac{c_{1} c_{2}}{c_{1}+c_{2}} \frac{\partial(q+r)}{\partial x_{2}}+\frac{\beta x_{2}\left(c_{1} q-c_{2} r\right)}{c_{1}+c_{2}}+\frac{c_{1}-c_{2}}{2\left(c_{1}+c_{2}\right)} \frac{\partial\left(c_{1} q-c_{2} r\right)}{\partial x_{2}}=0 .
\end{array}
$$

We have $r \equiv 0$, so the Rossby-gravity waves must satisfy

$$
\left(\frac{c_{1}+c_{2}}{2}\right) \frac{\partial u_{2}^{p}}{\partial x_{2}}+\beta x_{2} u_{2}^{p}=0
$$

which gives an expression for $u_{2}^{p}: u_{2}^{p}=A\left(x_{1}, t\right) \exp \left(-\frac{\beta}{c_{1}+c_{2}} x_{2}^{2}\right)$ and $q$ of the form $q=$ $B\left(x_{1}, t\right) x_{2} \exp \left(-\frac{\beta}{c_{1}+c_{2}} x_{2}^{2}\right)$, where $A$ and $B$ are linked as follows:

$$
\partial_{t} B+c_{1} \partial_{x_{1}} B=2 \beta A, \quad \partial_{t} A=-\frac{c_{1}}{2} B .
$$

We finally obtain an expression for the velocity field:

$$
u^{p}=\left(\begin{array}{c}
-\frac{2 \omega x_{2}}{c_{1}} \exp \left(\frac{-\beta}{c_{1}+c_{2}} x_{2}^{2}\right) \sin \left(k x_{1}-\omega t\right) \\
\exp \left(\frac{-\beta}{c_{1}+c_{2}} x_{2}^{2}\right) \sin \left(k x_{1}-\omega t\right)
\end{array}\right),
$$

with the dispersion relation: $\omega\left(\omega-k c_{1}\right)=\beta c_{1}$. These waves are dispersive, but, as Kelvin waves they are trapped in the equatorial waveguide.

We thus see that the new cosine terms give new velocities for equatorial waves that depend on the aspect ratio.

Let us now seek some equations (Quasi-Geostrophic, Lake) that are usually derived from the Shallow Water model. If we use the first order of the Shallow Water system, 
those equations remain unchanged. But we see in the following how the new terms modify those equations when we consider the second order system.

3. The quasi-geostrophic limit. The Quasi-Geostrophic model is used for modeling and forecasting mid-latitude oceanic and atmospheric circulation. It is obtained from the Shallow Water system if the Rossby and Froude numbers are small 1

We consider the dimensionless Shallow Water system (1.20)-(1.21). We write an asymptotic development $u=u^{0}+\eta u^{1}+\ldots, H=1+\eta F H^{1}+\ldots$ where we suppose $R o=\eta, F r^{2}=F \eta^{2}, b=\eta \tilde{b}$, with $\eta \ll 1, F=\left(2 \Omega L_{\text {char }}\right)^{2} /\left(g H_{\text {char }}\right)$ and $\varepsilon$ fixed.

We have to study the following equations:

$$
\begin{aligned}
\partial_{t} H+ & \operatorname{div}_{x}(H u)=0, \\
\partial_{t}(H u) & +\operatorname{div}_{x}(H u \otimes u)+\frac{1}{F \eta^{2}} H \nabla_{x} H \\
& =-\frac{\sin \theta}{\eta} H u^{\perp}+\varepsilon \frac{\cos \theta}{2 \eta} e_{1} H^{2} \operatorname{div}_{x} u+\varepsilon \frac{\cos \theta}{2 \eta} \nabla_{x}\left(H^{2} u_{1}\right) \\
& -\tilde{\alpha}_{0}(H) u+2 \nu \operatorname{div}_{x}(H D(u))+2 \nu \nabla_{x}(H \operatorname{div} u)+A H \nabla_{x} \Delta_{x} H \\
& +A H \nabla_{x} \Delta_{x} b-\frac{1}{F \eta^{2}} h \nabla_{x} b-\varepsilon \frac{\cos \theta}{\eta} H e_{1} \nabla_{x} b \cdot u+\varepsilon \frac{\cos \theta}{\eta} u_{1} H \nabla_{x} b .
\end{aligned}
$$

At the first and second orders, Equation (3.1) gives

$$
\operatorname{div}_{x} u^{0}=0 \quad \text { and } \quad F \partial_{t} H^{1}+\operatorname{div}_{x} u^{1}+F \nabla_{x} H^{1} \cdot u^{0}=0 .
$$

We also look at the first and second orders of the momentum equation (3.2). The first order gives

$$
\nabla_{x} H^{1}+\left(\sin \theta-\varepsilon \frac{\cos \theta}{2} \partial_{x_{2}}\right) u^{0^{\perp}}+\frac{\nabla_{x} \tilde{b}}{F}=0 .
$$

Then we take the $\operatorname{curl}$ (i.e., $-\partial_{x_{2}}$ of the first component and $+\partial_{x_{1}}$ of the second one) of the second order and get

$$
\begin{aligned}
\left(\partial_{t}+u^{0} \cdot \nabla_{x}\right) & \left(\operatorname{curl} u^{0}\right)=-\tilde{\alpha}_{0}(1) \operatorname{curl} u^{0}+\nu \Delta\left(\operatorname{curl} u^{0}\right) \\
& +\sin \theta F\left(\partial_{t} H^{1}+u^{0} \cdot \nabla_{x} H^{1}\right)-\sin \theta F u^{0} \cdot \nabla_{x} H^{1} \\
& +\varepsilon F \frac{\cos \theta}{2} \partial_{x_{2}}\left(\partial_{t} H^{1}+u^{0} \cdot \nabla_{x} H^{1}\right)-\nabla_{x}^{\perp} H^{1} \cdot \nabla_{x} \tilde{b} \\
& +\varepsilon \cos \theta \partial_{x_{2}}\left(u^{0} \cdot \nabla_{x} \tilde{b}\right)+\varepsilon \cos \theta \nabla_{x}^{\perp} u_{1}^{0} \cdot \nabla_{x} \tilde{b} .
\end{aligned}
$$

Let us remark that

$$
\sin \theta u^{0} \cdot \nabla_{x} H^{1}-\varepsilon \frac{\cos \theta}{2}\left(\partial_{x_{2}} u^{0}\right) \cdot \nabla_{x} H^{1}+\nabla_{x}^{\perp} H^{1} \cdot \frac{\nabla_{x} \tilde{b}}{F}=0
$$

and

$$
\left(\partial_{x_{2}} u^{0}\right) \cdot \nabla_{x} \tilde{b}+\nabla_{x}^{\perp} u_{1}^{0} \cdot \nabla_{x} \tilde{b}=0 .
$$

\footnotetext{
${ }^{1}$ Note that the Quasi-Geostrophic equations could also be obtained directly from the Navier-Stokes equations, considering the Rossby number comparable to the aspect ratio. This work is still under consideration.
} 
We define $\psi$ by $u^{0}=\nabla_{x}^{\perp} \psi$, which implies $H^{1}=\left(\sin \theta-\varepsilon \cos \theta \partial_{x_{2}} / 2\right) \psi-\tilde{b} / F$, and we find

$$
\begin{aligned}
\left(\partial_{t}+u^{0} \cdot \nabla_{x}\right)\left(\left(\partial_{x_{1}}^{2}+(1+\right.\right. & \left.\left.\varepsilon^{2} F \frac{\cos ^{2} \theta}{4}\right) \partial_{x_{2}}^{2}\right) \psi-\sin ^{2} \theta F \psi \\
& \left.+\left(\sin \theta-\varepsilon \frac{\cos \theta}{2} \partial_{x_{2}}\right) \tilde{b}\right)=-\tilde{\alpha}_{0}(1) \Delta \psi+\nu \Delta^{2} \psi .
\end{aligned}
$$

Equation (3.3) is the non-dimensional Quasi-Geostrophic equation obtained from the viscous Shallow Water system at the second order with a variable topography. The new terms due to the Coriolis force are $\varepsilon^{2} F \cos ^{2} \theta \partial_{x_{2}}^{2} \psi / 4$ and $\varepsilon \cos \theta \partial_{x_{2}} \tilde{b} / 2$. The "unusual" $\sin \theta$ coefficient is linked to an "unusual" Rossby number expression; in the "usual" case, the term $\sin \theta$ is replaced by 1 , and $\cos \theta$ by $1 / \tan \theta$. We could overcome this problem by writing the dimensional form of the Quasi-Geostrophic equation as in [7]. Note that the numerical experiments presented in this paper show that the cosine effect has a non-negligible influence for long times.

4. The Lake equations. We now consider other equations directly linked to the Shallow Water system: the Lake equations, where the domain is a shallow basin with a slowly spatially varying bottom. They are derived for a small Froude number.

To get the Lake equations we start with the second order Shallow Water system in non-dimensional form and we suppose there is no friction or viscosity:

$$
\begin{aligned}
& \partial_{t} H+\operatorname{div}(H u)=0, \\
& \begin{aligned}
& \partial_{t}(H u)+\operatorname{div}(H u \otimes u)+\frac{1}{F r^{2}} H \nabla H \\
&=-\frac{1}{F r^{2}} H \nabla b-\frac{\sin \theta}{R o} H u^{\perp}+\varepsilon \frac{\cos \theta}{2 R o} \nabla\left(u_{1} H^{2}\right) \\
&+\varepsilon \frac{\cos \theta}{2 R o} H^{2} e_{1} \operatorname{div} u-\varepsilon \frac{\cos \theta}{R o} H e_{1} \nabla b \cdot u+\varepsilon \frac{\cos \theta}{R o} u_{1} H \nabla b .
\end{aligned}
\end{aligned}
$$

Let $R o$ and $\varepsilon$ be fixed and $F r^{2}=\delta \ll 1$ : we are led to study the following equations:

$$
\begin{aligned}
& \partial_{t} H+\operatorname{div}(H u)=0, \\
& \begin{aligned}
& \partial_{t}(H u)+\operatorname{div}(H u \otimes u)+\frac{1}{\delta} H \nabla H \\
&=-\frac{1}{\delta} H \nabla b-\frac{\sin \theta}{R o} H u^{\perp}+\varepsilon \frac{\cos \theta}{2 R o} \nabla\left(u_{1} H^{2}\right) \\
&+ \varepsilon \frac{\cos \theta}{2 R o} H^{2} e_{1} \operatorname{div} u-\varepsilon \frac{\cos \theta}{R o} H e_{1} \nabla b \cdot u+\varepsilon \frac{\cos \theta}{R o} u_{1} H \nabla b .
\end{aligned}
\end{aligned}
$$

As we have done before, we develop the variables in powers of the small parameter $\delta$ : $u=u^{0}+\delta u^{1} \ldots, H=H^{0}+\delta H^{1} \ldots$

At the first order, the momentum equation (4.2) gives $H^{0} \nabla\left(H^{0}+b\right)=0$, so $H^{0}+b$ does not depend on spatial variables: $H^{0}+b=f(t)$.

Then we look at the first order of Equation (4.1): $f^{\prime}(t)+\operatorname{div}\left((f+b) u^{0}\right)=0$ and we integrate it in space considering periodic conditions. We get $f^{\prime}(t)=0$ and $H^{0}+b$ is a constant (in space and in time); we can suppose its value is equal to 1 . 
With this result, we can reformulate Equation (4.1):

$$
\operatorname{div}\left(H^{0} u^{0}\right)=0 \text {. }
$$

At the second order, Equation (4.2) gives

$$
\begin{aligned}
H^{0} \partial_{t} u^{0}+ & H^{0}\left(u^{0} \cdot \nabla\right) u^{0}+H^{0} \nabla H^{1}=-\frac{\sin \theta}{R o} H^{0} u^{0^{\perp}} \\
& +\varepsilon \frac{\cos \theta}{2 R o} H^{0}\left(H^{0} \nabla u_{1}^{0}+2 u_{1}^{0} \nabla H^{0}+H^{0} e_{1} \operatorname{div} u^{0}-2 e_{1} \nabla b \cdot u^{0}+2 u_{1}^{0} \nabla b\right) .
\end{aligned}
$$

Using the divergence relation (4.3), we remark that

$$
\begin{aligned}
\frac{1}{2} H^{0} \nabla u_{1}^{0}+u_{1}^{0} \nabla H^{0}+\frac{1}{2} H^{0} e_{1} \operatorname{div} u^{0}- & e_{1} \nabla b \cdot u^{0}+u_{1}^{0} \nabla b \\
& =\left(\partial_{x_{2}} H^{0}+\partial_{x_{2}} b+\frac{H^{0}}{2} \partial_{x_{2}}\right) u^{0^{\perp}}=\frac{H^{0}}{2} \partial_{x_{2}} u^{0^{\perp},}
\end{aligned}
$$

and we get

$$
\partial_{t} u^{0}+\left(u^{0} \cdot \nabla\right) u^{0}+\nabla P=-\left(\frac{\sin \theta}{R o}-\varepsilon \frac{\cos \theta}{2 R o} H^{0} \partial_{x_{2}}\right) u^{0^{\perp}}, \quad P=H^{1},
$$

which forms, with Equation (4.3), the Lake equations.

Now we want to express these equations in a vorticity formulation. We first recall how we get it in the "usual" case and then, we study our new term in $\cos \theta$.

The "usual" Lake equations are (4.3) and

$$
\partial_{t} u^{0}+\left(u^{0} \cdot \nabla\right) u^{0}+\nabla P+u^{0^{\perp}}=0, \quad \text { with } \operatorname{div}\left(H^{0} u^{0}\right)=0, \quad P=H^{1},
$$

and we know how to write it in vorticity formulation. We take the curl of the first equation and divide it by $H^{0}$. We use the equality $H^{0} \operatorname{div} u=-u \cdot \nabla H^{0}$ and we get

$$
\partial_{t} \frac{\operatorname{curl} u}{H^{0}}+\frac{\operatorname{curl}(u \cdot \nabla u)}{H^{0}}-\frac{u \cdot \nabla H^{0}}{H^{0^{2}}}=0 .
$$

We define $\Pi=\frac{1}{H^{0}} \operatorname{curl} u+\frac{1}{H^{0}}$ and we rewrite the system:

$$
\begin{aligned}
& \partial_{t} \Pi+u \cdot \nabla \Pi=0, \\
& u=\frac{1}{H^{0}} \nabla^{\perp} \psi .
\end{aligned}
$$

In our case, to get the vorticity formulation of the Lake equations (4.3)-(4.4), we have to look at the new term $H^{0} \partial_{x_{2}} u^{0^{\perp}}$.

With the divergence free condition, we find

$$
\begin{aligned}
\frac{1}{H^{0}} \operatorname{curl}\left(H^{0} \partial_{x_{2}} u^{0^{\perp}}\right) & =\frac{\nabla H^{0} \cdot \partial_{x_{2}} u}{H^{0}}+\operatorname{div}\left(\partial_{x_{2}} u_{2}\right) \\
& =\frac{\nabla H^{0} \cdot \partial_{x_{2}} u}{H^{0}}-\partial_{x_{2}}\left(\frac{u \cdot \nabla H^{0}}{H^{0}}\right) .
\end{aligned}
$$

Then, we write the relation

$$
\frac{1}{H^{0}} \operatorname{curl}\left(H^{0} \partial_{x_{2}} u^{0 \perp}\right)=-u \cdot \partial_{x_{2}}\left(\frac{\nabla H^{0}}{H^{0}}\right)=-u \cdot \nabla\left(\frac{\partial_{x_{2}} H^{0}}{H^{0}}\right)
$$


and we find a new system:

$$
\begin{aligned}
& \partial_{t} \Pi+u \cdot \nabla \Pi=0, \\
& u=\frac{1}{H^{0}} \nabla^{\perp} \psi, \\
& \text { where } \Pi=\frac{1}{H^{0}} \operatorname{curl} u+\frac{\sin \theta}{R o} \frac{1}{H^{0}}+\varepsilon \frac{\cos \theta}{2 R o} \frac{\partial_{x_{2}} H^{0}}{H^{0}}, \\
& \Pi_{\mid t=0}=\Pi_{0},
\end{aligned}
$$

which is the vorticity formulation of the Lake equations obtained from our new Shallow Water system.

5. If the latitude is not constant. At the beginning, we supposed the latitude to be constant. But we can do exactly the same development if the latitude is not constant. The only change is that we must keep the gradient of $\cos \theta u_{1} H^{2}$. We find the viscous Shallow Water system

$$
\begin{aligned}
& \partial_{t} H+\operatorname{div}_{x}(H u)=0, \\
& \partial_{t}(H u)+\operatorname{div}_{x}(H u \otimes u)+\frac{g}{2} \nabla_{x} H^{2}=-\alpha_{0}(H) u-\alpha_{1}(H) H u|u| \\
& \quad+a H \nabla_{x} \Delta_{x} H+2 \mu \nabla_{x}\left(H \operatorname{div}_{x} u\right)+2 \mu \operatorname{div}_{x}\left(H D_{x} u\right) \\
& \quad+\Omega \nabla_{x}\left(\cos \theta u_{1} H^{2}\right)+\Omega \cos \theta H^{2} e_{1} \operatorname{div}_{x} u-2 \Omega \sin \theta H u^{\perp} \\
& \quad-2 \Omega \cos \theta H e_{1} \nabla_{x} b \cdot u+2 \Omega \cos \theta u_{1} H \nabla_{x} b+a H \nabla_{x} \Delta_{x} b-g H \nabla_{x} b .
\end{aligned}
$$

We can also deduce from this system the Quasi-Geostrophic equation for a varying latitude. At the beginning, in dimensional variables, we replace $2 \Omega \sin \theta$ by $2 \Omega \sin \theta_{0}+\beta x_{2}$ and $2 \Omega \cos \theta$ by $2 \Omega \cos \theta_{0}-\beta \tan \theta_{0} x_{2}$ ( $\beta$-plane approximation, see [11]). We define a new non-dimensional parameter $\beta^{\prime}=\beta L_{\text {char }}^{2} / U_{\text {char }}$, and we find the non-dimensional Quasi-Geostrophic equation for a variable latitude:

$$
\begin{aligned}
\left(\partial_{t}+u^{0} \cdot \nabla_{x}\right)\left(\left(\partial_{x_{1}}^{2}\right.\right. & \left.+\left(1+\varepsilon^{2} F \frac{\cos ^{2} \theta_{0}}{4}\right) \partial_{x_{2}}^{2}\right) \psi-\sin ^{2} \theta_{0} F \psi \\
& \left.+\left(\sin \theta_{0}-\varepsilon \frac{\cos \theta_{0}}{2} \partial_{x_{2}}\right) \tilde{b}+\beta x_{2}\right)=-\tilde{\alpha}_{0}(1) \Delta \psi+\nu \Delta^{2} \psi .
\end{aligned}
$$

6. Mathematical properties of these models. In this part, we study mathematical properties of the previous models. Our goal is to establish the existence of global weak solutions for the Shallow Water system and for the Lake equations.

6.1. Existence of global solutions for shallow water equations. The study of the Shallow Water model has been done without the new Coriolis terms by F. MARCHE and P. FABRIE in [10] using Gronwall's lemma. We remark that the cosine effect involves derivatives (of order one in space) of the height and the velocity, and no control is $a$ priori available on them. Then we are led to use an appropriate interpolation lemma on these terms in order to be able to apply Gronwall's lemma in the same way as in [10.

The idea of the demonstration is first to get estimates thanks to the classical energy inequality and to a BD entropy type inequality. If we build sequences of approximate 
regular enough solutions as in [2], then we pass to the limit in the approximate problem and get the existence result.

REMARK 6.1. An important property to be noted is that our new Coriolis terms do not contribute in the energy estimates. Consequently, results based on this type of estimates remain unchanged.

6.1.1. Theorem. We consider the following Shallow Water system in a bounded domain $\mathcal{D}$ with periodic boundary conditions:

$$
\begin{aligned}
& \partial_{t} H+\operatorname{div}(H u)=0 \\
& \partial_{t}(H u)+\operatorname{div}(H u \otimes u)+\frac{1}{2 F r^{2}} \nabla H^{2}=-\tilde{\alpha}_{0}(H) u-\tilde{\alpha}_{1}(H) H u|u| \\
& \quad+A H \nabla \Delta H+A H \nabla \Delta b+\nu \operatorname{div}(H D(u))+\varepsilon \frac{\cos \theta}{2 R o} \nabla\left(u_{1} H^{2}\right) \\
& \quad+\varepsilon \frac{\cos \theta}{2 R o} H^{2} e_{1} \operatorname{div} u-\varepsilon \frac{\cos \theta}{R o} H e_{1} \nabla b \cdot u-\frac{\sin \theta}{R o} H u^{\perp} \\
& \quad-\nabla b\left(\frac{H}{F r^{2}}-\varepsilon \frac{\cos \theta}{R o} u_{1} H\right) .
\end{aligned}
$$

We suppose the bottom to be regular enough $\left(b \in H^{3}(\mathcal{D})\right)$ and the initial condition on the water height to satisfy: $H(t=0)=H_{0} \geq 0, \sqrt{H_{0}} \in H_{\text {per }}^{1}(\mathcal{D}), H_{0} \in L_{\text {per }}^{2}(\mathcal{D})$, $H_{0} u_{0}^{2} \in L_{\text {per }}^{1}(\mathcal{D}), \ln \left(K_{0}^{-1} H_{0} \tilde{\alpha}_{0}\left(H_{0}\right)\right) \in L_{\text {per }}^{1}(\mathcal{D})$. We prove the theorem:

Theorem 6.2. If $A>0, \tilde{\alpha}_{0}(H)>0$ and $\tilde{\alpha}_{1}(H) \geq 0$, with $H_{0}$ satisfying the above conditions, then there exists a global weak solution of (6.1).

\subsubsection{A priori estimates.}

Energy Inequality. The energy inequality associated to System (6.1) is classically given by

$$
\begin{gathered}
\frac{1}{2} \frac{d}{d t} \int_{\mathcal{D}}\left(\frac{H^{2}}{F r^{2}}+H|u|^{2}+A|\nabla H|^{2}\right)+\int_{\mathcal{D}} \tilde{\alpha}_{0}(H)|u|^{2}+\int_{\mathcal{D}} \tilde{\alpha}_{1}(H) H|u|^{3} \\
+\frac{\nu}{4} \int_{\mathcal{D}} H\left|\nabla u+{ }^{t} \nabla u\right|^{2} \leq \int_{\mathcal{D}} H\left(A \nabla \Delta b-\frac{\nabla b}{F r^{2}}\right) \cdot u
\end{gathered}
$$

In order to get better estimates, we write the BD entropy of our system. Entropy Inequality. 
Proposition 6.3. The entropy inequality for System (6.1) reads:

$$
\begin{aligned}
\frac{1}{2} \frac{d}{d t} & \int_{\mathcal{D}}\left(H|u+\nu \nabla \ln H|^{2}+\frac{H^{2}}{F r^{2}}+A|\nabla H|^{2}\right)+\frac{\nu}{4} \int_{\mathcal{D}} H\left|\nabla u-{ }^{t} \nabla u\right|^{2} \\
& -\nu K_{0} \frac{d}{d t} \int_{\mathcal{D}} \ln \left(K_{0}^{-1} H \tilde{\alpha}_{0}(H)\right)+\nu \int_{\mathcal{D}} \tilde{\alpha}_{0}^{\prime}(H) \nabla H \cdot u \\
& +\nu \int_{\mathcal{D}} \tilde{\alpha}_{1}(H)|u| u \cdot \nabla H+\nu A \int_{\mathcal{D}}\left|\nabla^{2} H\right|^{2}+\nu \int_{\mathcal{D}} \frac{|\nabla H|^{2}}{F r^{2}} \\
& +\nu \frac{\sin \theta}{R o} \int_{\mathcal{D}} u^{\perp} \cdot \nabla H-\nu \varepsilon \frac{\cos \theta}{2 R o} \int_{\mathcal{D}} e_{1} \cdot \nabla H H \operatorname{div} u \\
& -\nu \varepsilon \frac{\cos \theta}{2 R o} \int_{\mathcal{D}} \nabla\left(H^{2} u \cdot e_{1}\right) \cdot \nabla \ln H+\nu \varepsilon \frac{\cos \theta}{R o} \int_{\mathcal{D}} \nabla b \cdot u e_{1} \cdot \nabla H \\
& -\nu \varepsilon \frac{\cos \theta}{R o} \int_{\mathcal{D}} u \cdot e_{1} \nabla b \cdot \nabla H+\int_{\mathcal{D}} \tilde{\alpha}_{0}(H)|u|^{2}+\int_{\mathcal{D}} \tilde{\alpha}_{1}(H) H|u|^{3} \\
& \leq \int_{\mathcal{D}}\left(A \nabla \Delta b-\frac{\nabla b}{F r^{2}}\right) \cdot(H u+\nu \nabla H) .
\end{aligned}
$$

The proof relies on the following lemma:

LEMma 6.4. We have the following two equalities:

$$
\frac{1}{2} \frac{d}{d t} \int_{\mathcal{D}} H|\nabla \ln H|^{2}+\int_{\mathcal{D}} H \nabla \operatorname{div} u \cdot \nabla \ln H+\int_{\mathcal{D}} H \nabla u: \nabla \ln H \otimes \nabla \ln H=0
$$

and

$$
\begin{aligned}
\frac{1}{2} \nu^{2} & \frac{d}{d t} \int_{\mathcal{D}} H|\nabla \ln H|^{2}+\nu \int_{\mathcal{D}} \tilde{\alpha}_{0}(H) u \cdot \nabla \ln H+\nu \int_{\mathcal{D}} \tilde{\alpha}_{1}(H)|u| u \cdot \nabla H \\
& +\nu A \int_{\mathcal{D}}\left|\nabla^{2} H\right|^{2}+\nu \int_{\mathcal{D}} \frac{|\nabla H|^{2}}{F r^{2}}+\nu \frac{\sin \theta}{R o} \int_{\mathcal{D}} u^{\perp} \cdot \nabla H \\
& -\nu \varepsilon \frac{\cos \theta}{2 R o} \int_{\mathcal{D}} e_{1} \cdot \nabla H H \operatorname{div} u-\nu \varepsilon \frac{\cos \theta}{2 R o} \int_{\mathcal{D}} \nabla\left(H^{2} u \cdot e_{1}\right) \cdot \nabla \ln H \\
& =-\nu \frac{d}{d t} \int_{\mathcal{D}} u \cdot \nabla H+\nu \int_{\mathcal{D}} H \nabla u:{ }^{t} \nabla u-\nu \varepsilon \frac{\cos \theta}{R o} \int_{\mathcal{D}} \nabla b \cdot u e_{1} \cdot \nabla H \\
& +\nu \varepsilon \frac{\cos \theta}{R o} \int_{\mathcal{D}} u \cdot e_{1} \nabla b \cdot \nabla H+\nu \int_{\mathcal{D}}\left(A \nabla \Delta b-\frac{\nabla b}{F r^{2}}\right) \cdot \nabla H .
\end{aligned}
$$

REMARK 6.5. Our new terms only give us four terms in the second relation.

Proof. For the first equality, we refer to [1]. 
For the second one, we multiply the momentum equation by $\nu \nabla \ln H$ :

$$
\begin{gathered}
\nu \int_{\mathcal{D}}\left(\partial_{t} u+(u \cdot \nabla) u\right) \cdot \nabla H+\nu^{2} \int_{\mathcal{D}} D(u):\left(\nabla \nabla H-\frac{\nabla H \otimes \nabla H}{H}\right) \\
\quad+\nu A \int_{\mathcal{D}}\left|\nabla^{2} H\right|^{2}+\nu \int_{\mathcal{D}} \tilde{\alpha}_{0}(H) u \cdot \nabla \ln H+\nu \int_{\mathcal{D}} \tilde{\alpha}_{1}(H)|u| u \cdot \nabla H \\
\quad+\nu \int_{\mathcal{D}} \frac{|\nabla H|^{2}}{F r^{2}}+\nu \frac{\sin \theta}{R o} \int_{\mathcal{D}} u^{\perp} \cdot \nabla H-\nu \varepsilon \frac{\cos \theta}{2 R o} \int_{\mathcal{D}} e_{1} \cdot \nabla H H \operatorname{div} u \\
\quad-\nu \varepsilon \frac{\cos \theta}{2 R o} \int_{\mathcal{D}} \nabla\left(H^{2} u \cdot e_{1}\right) \cdot \nabla \ln H+\nu \varepsilon \frac{\cos \theta}{R o} \int_{\mathcal{D}} \nabla b \cdot u e_{1} \cdot \nabla H \\
-\nu \varepsilon \frac{\cos \theta}{R o} \int_{\mathcal{D}} u \cdot e_{1} \nabla b \cdot \nabla H=\nu \int_{\mathcal{D}}\left(A \nabla \Delta b-\frac{\nabla b}{F r^{2}}\right) \cdot \nabla H .
\end{gathered}
$$

We simplify this expression using

$$
\begin{gathered}
\int_{\mathcal{D}} H \nabla u: \nabla \ln H \otimes \nabla \ln H=\int_{\mathcal{D}} D(u): \frac{\nabla H \otimes \nabla H}{H}, \\
\int_{\mathcal{D}} D(u): \nabla \nabla H+\int_{\mathcal{D}} \nabla \operatorname{div} u \cdot \nabla H=0,
\end{gathered}
$$

and we add Equation (6.4) multiplied by $\nu^{2}$. We obtain:

$$
\begin{aligned}
\frac{1}{2} \nu^{2} \frac{d}{d t} \int_{\mathcal{D}} H|\nabla \ln H|^{2}+\nu \int_{\mathcal{D}} \frac{|\nabla H|^{2}}{F r^{2}}+\nu A \int_{\mathcal{D}}\left|\nabla^{2} H\right|^{2} \\
\quad+\nu \int_{\mathcal{D}} \tilde{\alpha}_{0}(H) u \cdot \nabla \ln H+\nu \int_{\mathcal{D}} \tilde{\alpha}_{1}(H)|u| u \cdot \nabla H+\nu \frac{\sin \theta}{R o} \int_{\mathcal{D}} u^{\perp} \cdot \nabla H \\
\quad-\nu \varepsilon \frac{\cos \theta}{2 R o} \int_{\mathcal{D}} e_{1} \cdot \nabla H H \operatorname{div} u-\nu \varepsilon \frac{\cos \theta}{2 R o} \int_{\mathcal{D}} \nabla\left(H^{2} u \cdot e_{1}\right) \cdot \nabla \ln H \\
\quad+\nu \varepsilon \frac{\cos \theta}{R o} \int_{\mathcal{D}} \nabla b \cdot u e_{1} \cdot \nabla H+\nu \varepsilon \frac{\cos \theta}{R o} \int_{\mathcal{D}} u \cdot e_{1} \nabla b \cdot \nabla H=I,
\end{aligned}
$$

where

$$
\begin{aligned}
& I=-\nu \int_{\mathcal{D}}\left(\partial_{t} u+(u \cdot \nabla) u\right) \cdot \nabla H+\nu \int_{\mathcal{D}}\left(A \nabla \Delta b-\frac{\nabla b}{F r^{2}}\right) \cdot \nabla H, \\
& I=-\nu \frac{d}{d t} \int_{\mathcal{D}} u \cdot \nabla H+\nu \int_{\mathcal{D}} H \nabla u:{ }^{t} \nabla u+\nu \int_{\mathcal{D}}\left(A \nabla \Delta b-\frac{\nabla b}{F r^{2}}\right) \cdot \nabla H .
\end{aligned}
$$


Proof of Proposition 6.3. We write Equation (6.5) as:

$$
\begin{aligned}
\frac{1}{2} \frac{d}{d t} \int_{\mathcal{D}} H|u+\nu \nabla \ln H|^{2}+\nu \int_{\mathcal{D}} \tilde{\alpha}_{0}(H) u \cdot \nabla \ln H \\
\quad+\nu \int_{\mathcal{D}} \tilde{\alpha}_{1}(H)|u| u \cdot \nabla H+\nu A \int_{\mathcal{D}}\left|\nabla^{2} H\right|^{2}+\nu \int_{\mathcal{D}} \frac{|\nabla H|^{2}}{F r^{2}} \\
\quad+\nu \frac{\sin \theta}{R o} \int_{\mathcal{D}} u^{\perp} \cdot \nabla H-\nu \varepsilon \frac{\cos \theta}{2 R o} \int_{\mathcal{D}} e_{1} \cdot \nabla H H \operatorname{div} u \\
\quad-\nu \varepsilon \frac{\cos \theta}{2 R o} \int_{\mathcal{D}} \nabla\left(H^{2} u \cdot e_{1}\right) \cdot \nabla \ln H+\nu \varepsilon \frac{\cos \theta}{R o} \int_{\mathcal{D}} \nabla b \cdot u e_{1} \cdot \nabla H \\
\quad-\nu \varepsilon \frac{\cos \theta}{R o} \int_{\mathcal{D}} u \cdot e_{1} \nabla b \cdot \nabla H \\
\quad=\frac{1}{2} \frac{d}{d t} \int_{\mathcal{D}} H|u|^{2}+\nu \int_{\mathcal{D}} H \nabla u:{ }^{t} \nabla u+\nu \int_{\mathcal{D}}\left(A \nabla \Delta b-\frac{\nabla b}{F r^{2}}\right) \cdot \nabla H
\end{aligned}
$$

and we add Equality (6.2) to get the result.

A priori Estimates. We consider $\left(H_{k}\right)_{k}$ and $\left(u_{k}\right)_{k}$ two sequences of weak solutions of (6.1) and satisfying (6.2) and (6.3). The energy inequality (6.2) classically gives the following estimates:

- $\left(H_{k}\right)_{k}$ in $L^{\infty}\left(0, T ; H_{p e r}^{1}(\mathcal{D})\right)$,

- $\left(\sqrt{H_{k}} u_{k}\right)_{k}$ in $L^{\infty}\left(0, T ;\left(L_{\text {per }}^{2}(\mathcal{D})\right)^{2}\right)$,

- $\left(\sqrt{H_{k}}\left(\nabla u_{k}+{ }^{t} \nabla u_{k}\right)\right)_{k}$ in $L^{2}\left(0, T ;\left(L_{p e r}^{2}(\mathcal{D})\right)^{4}\right)$,

- $\left(\tilde{\alpha}_{0}\left(H_{k}\right)^{1 / 2} u_{k}\right)_{k}$ in $L^{2}\left(0, T ;\left(L_{p e r}^{2}(\mathcal{D})\right)^{2}\right)$,

- $\left(\tilde{\alpha}_{1}\left(H_{k}\right)^{1 / 3} H_{k}{ }^{1 / 3} u_{k}\right)_{k}$ in $L^{3}\left(0, T ;\left(L_{p e r}^{3}(\mathcal{D})\right)^{2}\right)$.

We do not detail the proof here. We have more information thanks to the entropy inequality (6.3):

- $\left(\partial_{t} H_{k}\right)_{k}$ in $L^{\infty}\left(0, T ; W_{p e r}^{-1, q}(\mathcal{D})\right), \quad \forall q<2$,

- $\left(u_{k}\right)_{k}$ in $L^{2}\left(0, T ;\left(L_{\text {per }}^{2}(\mathcal{D})\right)^{2}\right)$,

- $\left(h_{k}^{1 / 3} u_{k}\right)_{k}$ in $L^{3}\left(0, T ;\left(L_{p e r}^{s}(\mathcal{D})\right)^{2}\right), \quad \forall s<3$,

- $\left(\tilde{\alpha}_{0}\left(H_{k}\right)\right)_{k}$ in $L^{\infty}\left(0, T ; H_{p e r}^{1}(\mathcal{D})\right)$,

- $\left(\tilde{\alpha}_{1}\left(H_{k}\right)\right)_{k}$ in $L^{\infty}\left(0, T ; H_{p e r}^{1}(\mathcal{D})\right)$,

- $\left(\nabla \sqrt{H_{k}}\right)_{k}$ in $L^{\infty}\left(0, T ;\left(L_{p e r}^{2}(\mathcal{D})\right)^{2}\right)$,

- $\left(H_{k}\right)_{k}$ in $L^{2}\left(0, T ; H_{p e r}^{2}(\mathcal{D})\right)$,

- $\left(\sqrt{H_{k}}\left(\nabla u_{k}-{ }^{t} \nabla u_{k}\right)\right)_{k}$ in $L^{2}\left(0, T ;\left(L_{\text {per }}^{2}(\mathcal{D})\right)^{4}\right)$ and consequently, with the results from the energy, $\left(\sqrt{H_{k}} \nabla u_{k}\right)_{k}$ in $L^{2}\left(0, T ;\left(L_{\text {per }}^{2}(\mathcal{D})\right)^{4}\right)$,

- $\left(H_{k} u_{k}\right)_{k}$ in $L^{2}\left(0, T ;\left(W_{p e r}^{1,1}(\mathcal{D})\right)^{2}\right)$.

Proof. For the first five estimates, we refer to [10]. The last ones are based on the entropy inequality (6.3), but are more intricate than without the cosine terms: we cannot directly write that $\left(\sqrt{H_{k}} \nabla u_{k}\right)_{k}$ is bounded in $L^{2}\left(0, T ;\left(L_{p e r}^{2}(\mathcal{D})\right)^{4}\right)$. We are led to combine terms on the right-hand side with terms on the left-hand side. 
From the results on $H_{k}$ and $u_{k}$, we know that $\left(H_{k} u_{k}\right)_{k}$ is in $L^{2}\left(0, T ;\left(L_{p e r}^{p}(\mathcal{D})\right)^{2}\right)$ for all $p<2$. We can also write:

$$
\begin{aligned}
&\left|\nu \int_{0}^{t} \int_{\mathcal{D}} \tilde{\alpha}_{1}\left(H_{k}\right) u\right| u \mid \cdot \nabla H_{k} \mid \\
& \leq C\left\|\sqrt{H_{k}} u_{k}\right\|_{L^{2}\left(0, T ;\left(L^{2}(\mathcal{D})\right)^{2}\right)}^{2}+\delta_{1}\left\|\sqrt{H_{k}} \nabla u_{k}\right\|_{L^{2}\left(0, T ;\left(L^{2}(\mathcal{D})\right)^{4}\right)}^{2} .
\end{aligned}
$$

So for small enough $\delta_{1}$ and for $\nu_{1}>0$, we can prove as in [10] that the following inequality holds for all $p<2$ :

$$
\begin{aligned}
& \frac{1}{2} \int_{\mathcal{D}} H_{k}\left|u_{k}+\nu \nabla \ln H_{k}\right|^{2}+\nu A \int_{0}^{t} \int_{\mathcal{D}}\left|\nabla^{2} H_{k}\right|^{2}+\nu_{1} \int_{0}^{t} \int_{\mathcal{D}} H_{k}\left|\nabla u-{ }^{t} \nabla u\right|^{2} \\
& \quad+\nu \frac{\sin \theta}{R o} \int_{0}^{t} \int_{\mathcal{D}} u_{k}{ }^{\perp} \cdot \nabla H_{k}-\nu \varepsilon \frac{\cos \theta}{2 R o} \int_{0}^{t} \int_{\mathcal{D}} e_{1} \cdot \nabla H_{k} H_{k} \operatorname{div} u_{k} \\
& \quad-\nu \varepsilon \frac{\cos \theta}{2 R o} \int_{0}^{t} \int_{\mathcal{D}} \nabla\left(H_{k}^{2} u_{k} \cdot e_{1}\right) \cdot \nabla \ln H_{k}+\nu \varepsilon \frac{\cos \theta}{R o} \int_{0}^{t} \int_{\mathcal{D}} \nabla b \cdot u_{k} e_{1} \cdot \nabla H_{k} \\
& \quad-\nu \varepsilon \frac{\cos \theta}{R o} \int_{0}^{t} \int_{\mathcal{D}} u_{k} \cdot e_{1} \nabla b \cdot \nabla H_{k} \leq C_{1}+C_{2}\left\|A \nabla \Delta b-\frac{\nabla b}{F r^{2}}\right\|_{\left(L^{2}(\mathcal{D})\right)^{2}} \\
& \quad \times\left(\left\|H_{k} u_{k}\right\|_{L^{2}\left(0, T ;\left(L^{p}(\mathcal{D})\right)^{2}\right)}+\left\|\nabla H_{k}\right\|_{L^{2}\left(0, T ;\left(L^{2}(\mathcal{D})\right)^{2}\right)}\right) .
\end{aligned}
$$

Then we have to study the Coriolis terms:

- $\left|\int_{0}^{t} \int_{\mathcal{D}} u_{k}^{\perp} \cdot \nabla H_{k}\right| \leq\left\|u_{k}\right\|_{L^{2}\left(0, T ;\left(L^{2}(\mathcal{D})\right)^{2}\right)}\left\|\nabla H_{k}\right\|_{L^{\infty}\left(0, T ;\left(L^{2}(\mathcal{D})\right)^{2}\right)}$,

- $\left|\int_{0}^{t} \int_{\mathcal{D}} e_{1} \cdot \nabla H_{k} H_{k} \operatorname{div} u_{k}\right|$

$$
\begin{aligned}
& \leq \int_{0}^{t}\left\|\nabla H_{k}\right\|_{\left(L^{2}(\mathcal{D})\right)^{2}}\left\|\sqrt{H_{k}}\right\|_{L^{\infty}(\mathcal{D})}\left\|\sqrt{H_{k}} \operatorname{div} u_{k}\right\|_{L^{2}(\mathcal{D})} \\
& \leq \frac{1}{4 \delta_{2}} \int_{0}^{t}\left\|\nabla H_{k}\right\|_{\left(L^{2}(\mathcal{D})\right)^{2}}^{2}\left\|\sqrt{H_{k}}\right\|_{L^{\infty}(\mathcal{D})}^{2}+\delta_{2} \int_{0}^{t}\left\|\sqrt{H_{k}} \operatorname{div} u_{k}\right\|_{L^{2}(\mathcal{D})}^{2} .
\end{aligned}
$$

For small enough $\delta_{2}$, the last term can be absorbed into the left-hand part. Let us look at the other term: with the estimates from the energy, we just have to control $\int_{0}^{t}\left\|\sqrt{H_{k}}\right\|_{L^{\infty}(\mathcal{D})}^{2}$. For all $\delta>0$, we can write:

$\left\|\sqrt{H_{k}}\right\|_{L^{\infty}(\mathcal{D})}^{2}=\left\|H_{k}\right\|_{L^{\infty}(\mathcal{D})} \leq\left\|H_{k}\right\|_{H^{1+\delta}(\mathcal{D})}$.

Thanks to Sobolev embedding, we get: $\left\|\sqrt{H_{k}}\right\|_{L^{\infty}(\mathcal{D})}^{2} \leq\left\|\nabla H_{k}\right\|_{L^{2 /(1-\delta)}(\mathcal{D})}$. Then we have the following inequalities:

$$
\begin{aligned}
\left\|\sqrt{H_{k}}\right\|_{L^{\infty}(\mathcal{D})}^{2} & \leq\left\|\nabla H_{k}\right\|_{L^{2 / 1-\delta}(\mathcal{D})} \leq C_{\delta}\left\|\nabla H_{k}\right\|_{L^{2}(\mathcal{D})}^{1-\delta}\left\|\nabla H_{k}\right\|_{H^{1}(\mathcal{D})}^{\delta} \\
& \leq C_{\delta}^{\prime}\left\|\nabla H_{k}\right\|_{L^{2}(\mathcal{D})}^{(2-2 \delta) /(2-\delta)}+\delta_{3}\left\|\nabla H_{k}\right\|_{L^{2}(\mathcal{D})}^{2}+\delta_{3}\left\|\Delta H_{k}\right\|_{L^{2}(\mathcal{D})}^{2} .
\end{aligned}
$$

So when we integrate in time, for small enough $\delta_{3}$, the last term can be taken up by the left-hand expression.

$$
\begin{aligned}
\text { - }\left|\int_{0}^{t} \int_{\mathcal{D}} \nabla\left(H_{k}^{2} u_{k} \cdot e_{1}\right) \cdot \nabla \ln H_{k}\right| \\
=\left|\int_{0}^{t} \int_{\mathcal{D}} 2 u_{k} \cdot e_{1}\left(\nabla H_{k}\right)^{2}+H_{k} \nabla H_{k} \cdot \nabla\left(u_{k} \cdot e_{1}\right)\right| .
\end{aligned}
$$

The second part can be controlled exactly as the previous term. For the first 
one, we write:

$\int_{\mathcal{D}} u_{k} \cdot e_{1}\left(\nabla H_{k}\right)^{2} \leq\left\|u_{k}\right\|_{L^{2}(\mathcal{D})}\left\|\nabla H_{k}\right\|_{L^{4}(\mathcal{D})}^{2}$.

As before, we know that $\left\|\nabla H_{k}\right\|_{L^{4}(\mathcal{D})}^{2} \leq C\left\|\nabla H_{k}\right\|_{L^{2}(\mathcal{D})}\left\|\nabla H_{k}\right\|_{H^{1}(\mathcal{D})}$.

Then we have:

$$
\begin{aligned}
\left|\int_{0}^{t} \int_{\mathcal{D}} 2 u_{k} \cdot e_{1}\left(\nabla H_{k}\right)^{2}\right| \leq C^{\prime}\left\|\nabla H_{k}\right\|_{L^{\infty}\left(0, T ; L^{2}(\mathcal{D})\right)}^{2}\left\|u_{k}\right\|_{L^{2}\left(0, T ; L^{2}(\mathcal{D})\right)}^{2} \\
+\delta_{4}\left\|\nabla H_{k}\right\|_{L^{2}\left(0, T ; L^{2}(\mathcal{D})\right)}^{2}+\delta_{4}\left\|\Delta H_{k}\right\|_{L^{2}\left(0, T ; L^{2}(\mathcal{D})\right)}^{2},
\end{aligned}
$$

and for a small $\delta_{4}$, the last term can be moved to the left.

$$
\begin{gathered}
\text { - }\left|\int_{0}^{t} \int_{\mathcal{D}} \nabla b \cdot u_{k} e_{1} \cdot \nabla H_{k}\right| \\
\quad \leq\|\nabla b\|_{\left(L^{\infty}(\mathcal{D})\right)^{2}}\left\|u_{k}\right\|_{L^{2}\left(0, T ;\left(L^{2}(\mathcal{D})\right)^{2}\right)}\left\|H_{k}\right\|_{L^{\infty}\left(0, T ; H^{1}(\mathcal{D})\right)}, \\
\text { - }\left|\int_{0}^{t} \int_{\mathcal{D}} u_{k} \cdot e_{1} \nabla b \cdot \nabla H_{k}\right| \\
\leq\|\nabla b\|_{\left(L^{\infty}(\mathcal{D})\right)^{2}}\left\|u_{k}\right\|_{L^{2}\left(0, T ;\left(L^{2}(\mathcal{D})\right)^{2}\right)}\left\|H_{k}\right\|_{L^{\infty}\left(0, T ; H^{1}(\mathcal{D})\right)} .
\end{gathered}
$$

Gathering all these relations, we get three new estimates on $\left(\nabla \sqrt{H_{k}}\right)_{k},\left(H_{k}\right)_{k}$ and on $\left(\sqrt{H_{k}} \nabla u_{k}\right)_{k}$.

The last one is finally obtained writing $\nabla\left(H_{k} u_{k}\right)$ as $\sqrt{H_{k}}\left(\sqrt{H_{k}} \nabla u_{k}\right)+u_{k} \cdot \nabla H_{k}$.

A Better Property for $H_{k} u_{k}$. In order to pass to the limit, we need better estimates on the product $H_{k} u_{k}$. We have:

Proposition 6.6. There exists a positive constant $C$ such that

$$
\left\|\tau_{\eta}\left(H_{k} u_{k}\right)-H_{k} u_{k}\right\|_{L^{\infty}\left(0, T-\eta ;\left(W_{\text {per }}^{1, \infty}(\mathcal{D})\right)^{\prime}\right.} \leq C \eta^{1 / 3},
$$

where $\tau_{\eta}$, for $\eta>0$ is the translation operator $\tau_{\eta} v(t, x)=v(t+\eta, x)$.

If for $1 \leq q \leq+\infty$ and $0<\zeta<1$, for a Banach space $E$ we denote by $N_{q}^{\zeta}(0, T ; E)$ the Nikolskii space defined as:

$$
N_{q}^{\zeta}(0, T ; E)=\left\{f \in L^{q}(0, T ; E) \text { s.t. } \exists C>0:\left\|\tau_{\eta} f-f\right\|_{L^{\infty}(0, T-\eta ; E)} \leq C \eta^{\zeta}\right\}
$$

this gives that $\left(H_{k} u_{k}\right)_{k}$ is bounded in $N_{\infty}^{1 / 3}\left(0, T ; W_{p e r}^{1, \infty}\right)$.

Proof. We multiply System (6.1) by a test function $\psi$ and we integrate over $\mathcal{D}$ and from $t$ to $t+\eta$. We find (see [10]):

$$
\begin{aligned}
& \left|\int_{\mathcal{D}}\left(\tau_{\eta}\left(H_{k} u_{k}\right)(t)-H_{k} u_{k}(t)\right) \psi\right| \leq\left(\int_{t}^{t+\eta} \mathrm{g}(s) d s\right)\|\psi\|_{W^{1, \infty}(\mathcal{D})} \\
& \quad+\frac{\sin \theta}{R o}\left|\int_{t}^{t+\eta} \int_{\mathcal{D}} H_{k} u_{k}^{\perp} \psi\right|+\frac{\varepsilon \cos \theta}{2 R o}\left|\int_{t}^{t+\eta} \int_{\mathcal{D}} H_{k}^{2} \operatorname{div} u_{k} e_{1} \psi\right| \\
& \quad+\frac{\varepsilon \cos \theta}{R o}\left|\int_{t}^{t+\eta} \int_{\mathcal{D}} H_{k} \nabla b \cdot u_{k} e_{1} \psi\right|+\frac{\varepsilon \cos \theta}{R o}\left|\int_{t}^{t+\eta} \int_{\mathcal{D}} H_{k}\left(u_{k}\right)_{1} \nabla b \psi\right| \\
& \quad+\frac{\varepsilon \cos \theta}{R o}\left|\int_{t}^{t+\eta} \int_{\mathcal{D}} H_{k}^{2}\left(u_{k}\right)_{1} \nabla \psi\right|
\end{aligned}
$$

with $\mathrm{g}$ bounded in $L^{3 / 2}(0, T)$. Let us consider the new terms: 


$$
\begin{aligned}
& \text { - }\left|\int_{\mathcal{D}} H_{k} u_{k}^{\perp} \psi\right| \leq\left\|H_{k} u_{k}\right\|_{L^{1}(\mathcal{D})}\|\psi\|_{L^{\infty}}, \\
& \text { • }\left|\int_{\mathcal{D}} H_{k}^{2} \operatorname{div} u_{k} e_{1} \psi\right| \leq\left\|H_{k}\right\|_{L^{6}(\mathcal{D})}\left\|\sqrt{H_{k}}\right\|_{L^{6}(\mathcal{D})}\left\|\sqrt{H_{k}} \operatorname{div}_{\mathrm{k}}\right\|_{L^{2}(\mathcal{D})}\|\psi\|_{L^{6}(\mathcal{D})}, \\
& \text { - }\left|\int_{\mathcal{D}} H_{k} \nabla b \cdot u_{k} e_{1} \psi\right| \leq\left\|H_{k} u_{k}\right\|_{L^{1}(\mathcal{D})}\|\nabla b\|_{L^{\infty}(\mathcal{D})}\|\psi\|_{L^{\infty}(\mathcal{D})}, \\
& \text { - }\left|\int_{\mathcal{D}} H_{k}\left(u_{k}\right)_{1} \nabla b \psi\right| \leq\left\|H_{k} u_{k}\right\|_{L^{1}(\mathcal{D})}\|\nabla b\|_{L^{\infty}(\mathcal{D})}\|\psi\|_{L^{\infty}(\mathcal{D})}, \\
& \text { - }\left|\int_{\mathcal{D}} H_{k}^{2}\left(u_{k}\right)_{1} \nabla \psi\right| \leq\left\|\sqrt{H_{k}} u_{k}\right\|_{L^{2}(\mathcal{D})}\left\|\sqrt{H_{k}}\right\|_{L^{8}(\mathcal{D})}\left\|H_{k}\right\|_{L^{8}(\mathcal{D})}\|\nabla \psi\|_{L^{4}(\mathcal{D})} .
\end{aligned}
$$

Then we can conclude that there exists $\tilde{g}$ bounded in $L^{3 / 2}(\mathcal{D})$ such that

$$
\left|\int_{\mathcal{D}}\left(\tau_{\eta}\left(H_{k} u_{k}\right)(t)-H_{k} u_{k}(t)\right) \psi\right| \leq\left(\int_{t}^{t+\eta} \tilde{\mathrm{g}}(s) d s\right)\|\psi\|_{W^{1, \infty}(\mathcal{D})},
$$

which gives

$$
\left\|\tau_{\eta}\left(H_{k} u_{k}\right)(t)-H_{k} u_{k}(t)\right\|_{\left(W_{p e r}^{1, \infty}(\mathcal{D})^{\prime}\right)} \leq \eta^{1 / 3}\|\tilde{\mathrm{g}}\|_{L^{3 / 2}(0, T)} .
$$

6.1.3. Convergence and compactness. We are now able to pass to the limit in the different terms. In [10], they proved that all the terms, except the ones linked to Coriolis, converge to the expected limit. Here, we study the other ones.

Properties on $H_{k}$ and $u_{k}$. First, we have $u_{k} \rightarrow u$ in $L^{2}\left(0, T ;\left(L_{p e r}^{2}(\mathcal{D})\right)^{2}\right)$.

About the sequence $\left(H_{k}\right)_{k}$, as we proved the inequalities $\left\|H_{k}\right\|_{L^{\infty}\left(0, T ; H^{1}(\mathcal{D})\right)} \leq C$ and $\left\|\partial_{t} H_{k}\right\|_{L^{\infty}\left(0, T ; H^{-2}(\mathcal{D})\right)} \leq C$, thanks to the compactness results [13] we find $H_{k} \rightarrow H$ in $\mathcal{C}^{0}\left(0, T ; H^{s}(\mathcal{D})\right)$, for all $s<1$.

Finally on the product $H_{k} u_{k}$, using both Proposition 6.6 and Sobolev properties, we get $H_{k} u_{k} \rightarrow H u$ in $L^{2}\left(0, T ;\left(L_{p e r}^{2}(\mathcal{D})\right)^{2}\right)$.

Study of the New Terms. For the usual Coriolis term, we can write, thanks to the above results, $H_{k} u_{k}^{\perp} \rightarrow H u^{\perp}$ in $L^{2}\left(0, T ;\left(L_{\text {per }}^{2}(\mathcal{D})\right)^{2}\right)$.

The terms with the bottom are also quickly treated:

$$
\begin{gathered}
H_{k} \nabla b \cdot u_{k} e_{1} \rightarrow H \nabla b \cdot u e_{1} \text { in } L^{2}\left(0, T ;\left(L_{p e r}^{2}(\mathcal{D})\right)^{2}\right), \\
\text { and } \quad H_{k}\left(u_{k}\right)_{1} \nabla b \rightarrow H u_{1} \nabla b \text { in } L^{2}\left(0, T ;\left(L_{p e r}^{2}(\mathcal{D})\right)^{2}\right) .
\end{gathered}
$$

The last two terms read:

$$
\left(u_{k}\right)_{1} H_{k}^{2} \rightarrow u_{1} H^{2} \text { in } L^{1}\left(0, T ;\left(L^{1}(\mathcal{D})\right)^{2}\right),
$$

and, as $\sqrt{H_{k}} \operatorname{div} u_{k} \rightarrow \sqrt{H} \operatorname{div} u$ in $L^{2}\left(0, T ; L^{2}(\mathcal{D})\right)$ and $H_{k} \rightarrow H$ in $\mathcal{C}^{0}\left(0, T ; H^{s}(\mathcal{D})\right)$, for all $s<1$, we get

$$
H_{k}^{2} \operatorname{div} u_{k} e_{1} \rightarrow H^{2} \operatorname{div} u e_{1} \text { in } L^{1}\left(0, T ; L^{1}(\mathcal{D})\right) .
$$

6.1.4. End of the proof. We have proved that from a sequence verifying Equations (6.2) and (6.3) we can extract a subsequence that strongly converges to a weak solution of (6.1). As in 2, we can construct sequences of approximate smooth enough solutions of (6.1) using a regularization process. Considering such sequences, we classically get the existence result. 
6.2. Existence of a solution for the Lake equations. Just as in the Shallow Water system case, the existence of a solution for the Lake equations has been proved in [5] in the non-degenerate case, and in [3] for the degenerate case, without the term in $\cos \theta$.

We see here how to adapt these proofs to our case.

6.2.1. If $H^{0}$ does not vanish. We rewrite the vorticity formulation as

$$
\begin{aligned}
& \partial_{t} \Pi+u \cdot \nabla \Pi=0, \\
& u=\tilde{K} \Pi, \\
& \Pi_{\mid t=0}=\Pi_{0} .
\end{aligned}
$$

The operator $\tilde{K}$ is a linear operator such that $u$ satisfies $\operatorname{div}\left(h^{0} u\right)=0$. It can be seen as a perturbation of the inverse of the operator curl.

In [5], C. D. Levermore, M. Oliver and E. S. Titi consider the following system:

$$
\begin{aligned}
& \partial_{t} \Pi+u \cdot \nabla \Pi=0, \\
& u=K \Pi, \\
& \Pi_{\left.\right|_{t=0}}=\Pi_{0},
\end{aligned}
$$

in a domain $\mathcal{D}$, where $K$ is a linear operator such that we have the condition $\operatorname{div}\left(h^{0} u\right)=0$.

In our case, the operator $\tilde{K}$ can be deduced from the operator $K$ because $\tilde{K} \Pi$ is the sum of $K \Pi$ and terms that do not depend on the unknowns. Then the estimate on $K$ is modified in

$$
\|\tilde{K} \Pi\|_{W^{1, p}} \leq c p\|\Pi\|_{L^{p}}+F
$$

where $F$ only depends on $\theta, \varepsilon$ and $H^{0}$.

We can do the same reasoning: we first consider the problem with artificial viscosity

$$
\partial_{t} \Pi_{\nu}+u \cdot \nabla \Pi_{\nu}-\nu H^{-1} \nabla \cdot\left(H \nabla \Pi_{\nu}\right)=0 .
$$

If the initial value of $\Pi_{\nu}$ is in $H_{0}^{1}(\mathcal{D})$, we can prove there exists a unique solution $\Pi_{\nu}$ in $\mathcal{C}^{0}\left(\left[0,+\infty\left[, H_{0}^{1}(\mathcal{D})\right) \cap L_{\text {loc }}^{2}\left(\left[0,+\infty\left[, H^{2}(\mathcal{D}) \cap H_{0}^{1}(\mathcal{D})\right)\right.\right.\right.\right.$ for each $\nu$. This result is obtained by construction of a family of approximate solutions (solutions in the subset spanned by the first $n$ eigenvectors) and we pass to the limit thanks to compactness.

Then we study the inviscid limit: we have compactness of the sequence $\Pi_{\nu}$, which enables us to pass to the limit and claim that if the initial value is in $L^{\infty}(\mathcal{D})$, there exists $\Pi$ in $\mathcal{C}^{0}\left(\left[0,+\infty\left[, \mathrm{w}^{\star}-L^{\infty}\left(\left[0,+\infty[\times \mathcal{D}) \cap L^{\infty}([0,+\infty[\times \mathcal{D}))\right.\right.\right.\right.\right.$.

In the study of the uniqueness, the new Coriolis terms do not appear because we compute the difference between two solutions.

We can thus conclude that, if the initial vorticity $\Pi_{0}$ is in $L^{\infty}(\mathcal{D})$, we have existence and uniqueness of global solutions to the Lake equations.

6.2.2. If $H^{0}$ vanishes. This part is directly linked to the estimates proved by BRESCH and MÉTIVIER in [3] for the system

$$
\begin{aligned}
& \operatorname{div}\left(H^{0} u\right)=0, \\
& \operatorname{curl} u=f,
\end{aligned}
$$

where $f$ is in $L^{p}(\mathcal{D})$ and $H^{0} u$ is in $L^{2}(\mathcal{D})$.

So with the same arguments we can deduce estimates on the solution. 
About the existence of global solutions, the use of an artificial viscosity can also be adapted: we define $H_{\nu}^{0}=H^{0}+\nu$ and we change the Lake equations for $\partial_{t}\left(H_{\nu}^{0} \Pi_{\nu}\right)+$ $H_{\nu}^{0} u_{\nu} \cdot \nabla \Pi_{\nu}-\nu \operatorname{div}\left(H_{\nu}^{0} \nabla \Pi_{\nu}\right)=0$. But the new terms of our model (compared to [3]) only add the $x_{2}$ derivative of $H_{\nu}^{0}$; so we pass to the limit in the same way.

The proof of uniqueness is based on Yudovitch's work: let $u_{1}$ and $u_{2}$ be two solutions of the Lake equations

$$
\partial_{t} u_{1 / 2}+u_{1 / 2} \cdot \nabla u_{1 / 2}+\nabla P_{1 / 2}=-\left(\frac{\sin \theta}{R o}-\varepsilon \frac{\cos \theta}{2 R o} H \partial_{x_{2}}\right) u_{1 / 2}^{\perp} .
$$

The function $u$ defined by $u=u_{1}-u_{2}$ is solution of

$$
\partial_{t} u+u \cdot \nabla u_{1}+u_{2} \cdot \nabla u+\nabla P=-\left(\frac{\sin \theta}{R o}-\varepsilon \frac{\cos \theta}{2 R o} H \partial_{x_{2}}\right) u^{\perp} .
$$

We multiply by $H u$ and we get

$$
\frac{1}{2} \frac{d}{d t} \int H|u|^{2}+\int u \cdot \nabla u_{1} H u=0 .
$$

As we have

$$
\begin{aligned}
\left|\int u \cdot \nabla u_{1} H u\right| & \leq\|\sqrt{H} u\|_{L^{\infty}}^{\frac{2}{p}}\left(\|\sqrt{H} u\|_{L^{2}}^{2}\right)^{1-\frac{1}{p}}\left\|\nabla u_{1}\right\|_{L^{p}} \\
& \leq\|\sqrt{H} u\|_{L^{\infty}}^{\frac{2}{p}}\left(\|\sqrt{H} u\|_{L^{2}}^{2}\right)^{1-\frac{1}{p}}(p C+F),
\end{aligned}
$$

we get the following inequality:

$$
\frac{d}{d t}\|\sqrt{H} u\|_{L^{2}}^{2} \leq\left(\|\sqrt{H} u\|_{L^{2}}^{2}\right)^{1-\frac{1}{p}}(p C+F) M,
$$

which gives $u=0$.

So we conclude with the theorem:

Theorem 6.7. For all initial data $\Pi_{0} \in L^{\infty}(\mathcal{D})$ there exists a unique weak solution $(u, \Pi)$ for the Lake equations, with $\Pi$ belonging to $\mathcal{C}^{0}\left([0, T] ; L^{\infty}(\mathcal{D})\right.$ weak $\left.\star\right)$, verifying $\Pi / H^{0}$ in $L^{\infty}([0, T] \times \mathcal{D})$ and $H^{0} u$ in $\mathcal{C}^{0}\left([0, T] ; L^{2}(\mathcal{D})\right)$.

Furthermore, this solution is regular, that is, $\Pi / h^{0}$ is in $\mathcal{C}^{0}\left([0, T] ; L^{r}(\mathcal{D})\right)$ and $u$ is in $\mathcal{C}^{0}\left([0, T] ; W^{1, r}(\mathcal{D})\right)$ for all finite $r$.

Acknowledgments. The author would like to thank D. BRESCH for fruitful discussions.

\section{REFERENCES}

[1] D. Bresch and B. Desjardins. Existence of global weak solutions for a 2D viscous shallow water equations and convergence to the quasi-geostrophic model, Comm. Math. Phys., 238 1-2 (2003), 211-223. MR.1989675 (2004d:76026)

[2] D. Bresch and B. Desjardins. On the construction of approximate solutions for the 2D viscous Shallow Water model and for compressible Navier-Stokes models, J. Math. Pure Appl., 86 (2006), 362-368. MR 2257849 (2007j:35161)

[3] D. Bresch and G. Métivier. Global existence and uniqueness for the lake equations with vanishing topography: elliptic estimates for degenerate equations, Nonlinearity, 193 (2006), 591-610. MR2209290 (2007b:35261) 
[4] J.-F. Gerbeau and B. Perthame. Derivation of viscous Saint-Venant system for laminar shallow water; numerical validation, Discrete and Continuous Dynamical Systems-series B., 11 (2001), 89-102. MR:1821555 (2001m:76029)

[5] C. D. Levermore, M. Oliver and E. S. Titi. Global well-posedness for models of shallow water in a basin with a varying bottom, Indiana Univ. Math. J, 45 (1996), 479-510. MR1414339 (97m:35214)

[6] C. Lucas. Effet cosinus sur un modèle visqueux de type Saint-Venant et ses équations limites de type quasi-géostrophique et lacs, C. R. Acad. Sci. Paris, Ser. I, 3456 (2007), 313-318. MR2359088 (2008h:35294)

[7] C. Lucas and A. Rousseau. New Developments and Cosine Effect in the Viscous Shallow Water and Quasi-Geostrophic Equations, SIAM Multiscale Modeling and Simulation, 72 (2008), 796-813.

[8] A. Majda. Introduction to PDEs and Waves for the Atmosphere and Ocean, Courant Lecture Notes in Math. 9, American Mathematical Society (2003). MR.1965452 (2004b:76152)

[9] F. Marche. Theoretical and numerical study of shallow water models; applications to nearshore hydrodynamics, Ph.D., Université Bordeaux (2005).

[10] F. Marche and P. Fabrie. Another proof of stability for global weak solutions of $2 D$ degenerated Shallow Water models, Journal of Mathematical Fluid Mechanics, (2008).

[11] J. Pedlosky. Geophysical fluid dynamics, 2d edition, Springer (1987).

[12] A.J.-C. de Saint-Venant. Théorie du mouvement non-permanent des eaux, avec application aux crues des rivières et à l'introduction des marées dans leur lit, Comptes Rendus de l'Académie des Sciences 73 (1871), 147-154.

[13] J. Simon. Compact Sets in the Space $L^{p}(0, T ; B)$, Ann. Mat. Pura Appl., 1464 (1987), 65-96. MR.916688 (89c:46055) 